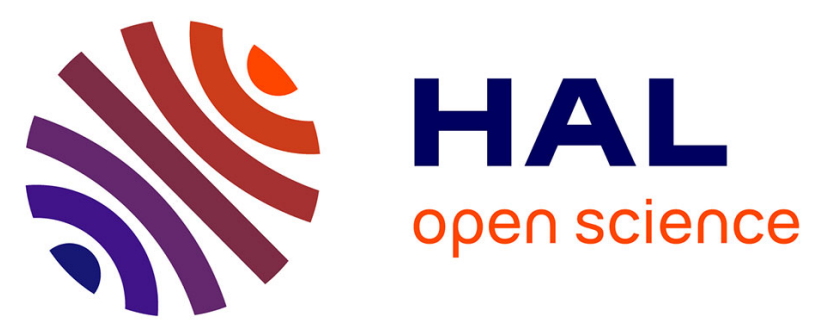

\title{
Former des enseignants de langue étrangère et seconde aux dimensions interculturelles explicites en France et en Colombie. Un dialogue théorique et épistémologique
} Paola Gamboa Diaz, Muriel Molinié, Isabel Tejada Sanchez

\section{- To cite this version:}

Paola Gamboa Diaz, Muriel Molinié, Isabel Tejada Sanchez. Former des enseignants de langue étrangère et seconde aux dimensions interculturelles explicites en France et en Colombie. Un dialogue théorique et épistémologique. Interculturalidad y formación de profesores. Perspectivas pedagógicas y multilingües. Bogota. Ediciones Uniandes, pp.3-42, 2019, 978-958-774-845-1. hal-02560480

\author{
HAL Id: hal-02560480 \\ https://hal.science/hal-02560480
}

Submitted on 1 May 2020

HAL is a multi-disciplinary open access archive for the deposit and dissemination of scientific research documents, whether they are published or not. The documents may come from teaching and research institutions in France or abroad, or from public or private research centers.
L'archive ouverte pluridisciplinaire $\mathbf{H A L}$, est destinée au dépôt et à la diffusion de documents scientifiques de niveau recherche, publiés ou non, émanant des établissements d'enseignement et de recherche français ou étrangers, des laboratoires publics ou privés. 
Gamboa Diaz, P. Molinié, M., \& Tejada-Sánchez, I. (2019) Former des enseignants de langue étrangère et seconde aux dimensions interculturelles explicites en France et en Colombie. Un dialogue théorique et épistémologique in B. Peña-Dix, I. Tejada-Sanchez, \& A. Truscott de Mejia. Interculturalidad y formación de profesores. Perspectivas pedagógicas y multilingües (ISBN 978-958-774-845-1), pp. 3-42. Bogotá: Ediciones Uniandes.

\title{
1 \\ Former des enseignants de langue étrangère et seconde aux dimensions interculturelles explicites en France et en Colombie : un dialogue théorique et épistémologique
}

\author{
Paola Gamboa-Diaz \\ Muriel Molinié \\ ISABEL TEJADA-SÁNCHEZ
}

Contexte global, national et local : transformations

socio-éducatives et enseignement des langues

Comme l'expose Gürüz (2011), la globalisation économique est l'un des facteurs à l'origine de l'internationalisation de l'enseignement supérieur qui construit des offres de formation aux formes multiples, avec en particulier des modalités en présence, à distance ou hybrides. En effet, les institutions et organismes fournisseurs de services éducatifs se voient dans l'obligation de répondre aux demandes des gouvernements qui, eux-mêmes, font le choix politique de rendre leur pays compétitif dans le domaine de l'économie des savoirs: «... the country's capacity to take advantage of the global knowledge economy ... depends on its capacity to participate, at least to some extent, in the processes of generating, accessing and sharing knowledge ». Gürüz (2011, p. 18).

* Pour citer ce chapitre : http://dx.doi.org/10.30778/2019.58 
Les partenariats internationaux, nationaux et locaux entre universités se sont intensifiés et avec ceux-ci, les mobilités académiques. Dans ce contexte mondial, l'enseignement-apprentissage des langues secondes et étrangères prend une place considérable dans la mesure où ces langues deviennent vectrices d'accès à la mobilité académique et subséquemment à la mobilité dans le monde du travail. Nos deux pays, la France et la Colombie, se sont inscrits dans une dynamique économique fondée sur le savoir et le développement de l'enseignement supérieur. L'accès aux savoirs universitaires joue donc un rôle clé dans le développement économique des deux pays.

C'est ainsi qu'en Colombie el Ministerio de Educación Nacional (MEN) a mis en place depuis 2004, un programme national d'éducation bilingue espagnol-anglais, dans le but de rendre l'ensemble des citoyens plus compétents en langues étrangères. De l'école primaire à la fin du lycée, les élèves doivent avoir un bon niveau en anglais et, pendant le cursus universitaire, les étudiants doivent atteindre un très bon niveau de langue. En France chaque élève doit être capable de communiquer dans au moins deux langues vivantes à la fin du secondaire. Pour atteindre cet objectif, l'enseignement des langues s'inscrit dans une perspective européenne. Les élèves sont sensibilisés à une langue étrangère dès le cours préparatoire, l'apprentissage et la pratique d'autres langues étrangères ou secondes sont progressivement introduits du collège au lycée.

Mais si les institutions éducatives se trouvent confrontées aux besoins socio-économiques évoqués ci-dessus, celles-ci sont également conduites à prendre en charge des réalités linguistiques et culturelles multiples: ainsi sur le territoire colombien ${ }^{1}$ on trouve des situations de bi-multi-plurilinguisme avec 68 langues indigènes, 2 créoles, 1 langue Rom, 1 langue de signes colombienne, l'anglais de San Andrés et Providencia et le castillan (ou espagnol) comme langues officielles. En France, on répertorie plus de 10 langues régionales, plus de 8 créoles et langues autochtones parlées dans les collectivités d'outre-mer, la langue de signes française et le français en qualité de langue nationale ${ }^{2}$ sans oublier l'ensemble des langues « non-territoriales » parlées par les migrants et leurs descendants. Tout ceci fait que « par ordre d'importance, les langues

1 Portal de Lenguas de Colombia. En ligne http://lenguasdecolombia.caroycuervo.gov.co/, consulté le 14/08/2017. Constitución Política de Colombia (1991). Título 3. Artículo 10. El castellano es el idioma oficial de Colombia. Las lenguas y dialectos de los grupos étnicos son también oficiales en sus territorios. La enseñanza que se imparta en las comunidades con tradiciones lingüísticas propias será bilingüe. En ligne http://www.constitucioncolombia.com/titulo-1/ capitulo-o/articulo-10, consulté le 1/08/2017.

2 Anciens département et/ ou région d'outre-mer (DOM-ROM), départements d’outre-mer (DOM); territoires d'outre-mer (том), appelés en 2015 collectivités d'outre-mer (сом). L’aménagement linguistique dans le monde. République française. En ligne http://www.axl.cefan.ulaval.ca/europe/ france.htm, consulté le 14/08/2017. 
les plus pratiquées en France en dehors du français, selon l'ined, sont l'arabe dialectal ( 3 ou 4 millions de locuteurs), les créoles et le berbère (près de deux millions), l'alsacien (548 ooo), l'occitan (526 ooo), le breton (304 ooo), les langues d'oïl (204 00o), le francique mosellan ou platt lorrain (78 ooo), le corse (60 ooo), le basque (44 00o) $»^{3}$.

\section{Contextes de la recherche}

\section{L'enseignement de langues secondes et étrangères en Colombie}

Lorsqu'on parle des langues en Amérique Latine, les réalités linguistiques et éducatives sont intimement liées. D’une part, il est question de la diversité linguistique et culturelle léguée par les peuples autochtones; d'autre part, il est question de l'influence des économies étrangères et de leurs langues majoritaires dans l'agenda politique et social de la région.

En Colombie, cette réalité est incarnée par la coexistence et la reconnaissance constitutionnelle des langues minoritaires et des langues internationales majoritaires, à savoir le castillan ou espagnol, langue officielle, et l'anglais, la langue étrangère la plus populaire du pays (De Mejía, 2005).

Par ailleurs, la situation sociolinguistique de la Colombie est complexifiée et menacée, davantage, à cause du conflit armé et du déplacement des populations rurales et indigènes qui dure depuis plusieurs décennies, malgré la signature d'un accord de paix avec l'un des acteurs armés. Cependant, des efforts de préservation, de revitalisation et de valorisation des patrimoines immatériels se déploient de plus en plus au niveau du gouvernement national et dans la sphère privée. En outre, dans l'esprit de ce que l'on appelle le post-conflit, des initiatives intéressantes ont vu le jour dans les domaines de la recherche, de la cohésion sociale et de l'éducation notamment.

Dans le domaine de l'éducation et de la transmission de langues indigènes, le gouvernement en s'appuyant sur la Constitution de 1991 et sur des lois des langues comme la loi $1381^{[4]}$ de 2010 , promeut, l'autonomie des régions dans

3 http://www.culturecommunication.gouv.fr/Thematiques/Langue-francaise-et-langues-deFrance/Politiques-de-la-langue/Langues-de-France.

4 Ley de Protección de las Lenguas Nativas: "Por la cual se desarrollarán los artículos $7^{\circ}, 8^{\circ}, 10$ y 70 de la constitución política, y los artículos $4^{\circ}, 5^{\circ}$ y 28 de la Ley 21 de 1991 (que aprueba el convenio 169 de la oIT sobre pueblos indígenas y tribales), y se dictan normas sobre reconocimiento; fomento, protección, uso, preservación y fortalecimiento de las lenguas de los grupos étnicos de Colombia y sobre sus derechos lingüísticos y los de sus hablantes". Portal Lenguas de Colombia. En ligne http:// lenguasdecolombia.caroycuervo.gov.co/documentos/Ley_1381_2010_proteccion_lenguas_nativas. pdf, consulté le 1/08/2017. 
leur gestion des langues autochtones qui y sont parlées. Par exemple, l'initiative Etnoeducación (MEN, 2001) a été conçue dans le but de soutenir les communautés des langues autochtones. Dans ce système, l'enfant aurait accès à un enseignement qui tient compte de ses langues et ses cultures autochtones ainsi que de la langue nationale et de la langue étrangère. Cependant, ce programme demeure une préconisation institutionnelle et les initiatives d'enseignement systématique de ces langues sont encore isolées. Parmi les expériences isolées, celle de Jaraba et Arrieta, (2012) essaie d'inclure trois langues dans l'enseignement public: l'espagnol, une langue autochtone et l'anglais. De manière plus générale, certains programmes de recherche constituent des bases fondamentales pour la réflexion dans ce domaine. Par exemple, à l'Université del Cauca, le Grupo de Estudios lingüísticos, pedagógicos y socioculturales (GELPS) ${ }^{5}$ dirigé par le professeur Tulio Rojas Curieux, possède une longue expérience dans la recherche et l'élaboration d'outils pédagogiques en Nasa- Yuwe et Nam Trik, deux langues autochtones du sud-ouest de la Colombie. D’autres initiatives constituent le projet Martínez Sierra, Naranjo Cuervo et Rojas Curieux (2010) sur la coopération entre ethno-éducation et nouvelles technologies. Malgré ces travaux, l'éducation en langues minoritaires reste une tradition communautaire encore rarement institutionnalisée et, en conséquence, un domaine encore cloisonné. Il en découle que la plupart des communautés amérindiennes et créoles suivent encore leur scolarisation avec « un programme presque entièrement en espagnol » (De Mejía, 2005, p. 385).

En revanche, pour ce qui est des langues majoritaires et internationales, l'enseignement d'une langue étrangère $(\mathrm{LE})$ ou langue seconde ( $\mathrm{L} 2)$ s'avère un enjeu fondamental, notamment depuis le début du $21^{\mathrm{e}}$ siècle en Colombie. Cette dynamique s'illustre en particulier par la notoriété des termes « enseignement bilingue » et "bilinguisme » dans l'agenda politique, éducatif, académique et social de ce pays (Bonilla \& Tejada-Sánchez, 2016; De Mejía, 2013; Truscott de Mejía, López Mendoza \& Peña Dix, 2011). Il faut noter que désormais, cette tendance favorise surtout l'enseignement de l'anglais à l'école. La situation géopolitique et socioéconomique de la Colombie et le cadre d'une relation binationale proche avec les Etats-Unis, qui date de deux siècles déjà (Randall, 1992), impulse l'apprentissage de l'anglais associé à une culture de la compétitivité. En effet, étant donné que la Colombie maintient des relations sociopolitiques et économiques importantes avec les Etats-Unis, elle se voit très fortement influencée par la culture et le commerce nord-américains. Ces facteurs rendent le phénomène

5 Site du groupe de recherche « Grupo de estudios lingüísticos pedagógicos y socioculturales » à l'Université du Cauca, en Colombie. En ligne http://www.unicauca.edu.co/gelps/es/inicio, consulté le $1 / 08 / 2017$. 
de l'anglais comme lingua franca ou langue internationale (ELF \& EIL) (Jenkins, 2006) très visible dans ce contexte.

L'on constate également que l'enseignement des langues en Colombie donne lieu à deux réalités distinctes. D'une part, l'enseignement bilingue fait plutôt référence aux langues majoritaires et, par conséquent, le bilinguisme renvoie principalement au binôme espagnol/langue internationale (anglais, dans la plupart de cas). Ce qui signifie que le bilinguisme est rarement associé à la réalité des peuples autochtones (amérindiens, afrodescendants, Rom) du pays. D'autre part, on observe un écart entre les systèmes d'éducation public et privé dans le domaine des langues étrangères. De ce fait, depuis la mise en place du Programme National de Bilinguisme en 2004 et des lois subséquentes (Bonilla \& Tejada-Sánchez, 2016), le gouvernement essaie de promouvoir des standards de compétence en anglais comparables entre les établissements publics et privés, en suivant surtout les orientations du cadre européen commun de références pour les langues (CECRL).

Ainsi, des écoles privées peuvent adopter des modèles d'enseignement de langues selon leurs intérêts, tout en respectant le reste du programme éducatif national établi par le ministère de l'éducation. Par rapport au secteur public, le programme récemment renommé Colombia Bilingue (MEN, 2015) a promu des actions concrètes, à savoir:

- La formulation de directives adressées aux écoles publiques et privées pour le curriculum d'anglais langue étrangère (lineamientos curriculares en inglés).

- La mise en place de programmes d'immersion pour les enseignants en exercice, ainsi que la promotion de bourses et de crédits pour continuer des études dans l'enseignement supérieur, afin de fournir aux enseignants des opportunités d'approfondir leurs connaissances de la L2/ LE et de la didactique.

- L'arrivée d'assistants de langue anglaise afin de promouvoir la communication orale.

- La création et mise à disposition de matériel technologique pour l'enseignement et l'apprentissage de l'anglais.

Suite à la formulation d'une telle politique éducative dans le domaine des langues étrangères, trois modèles d'enseignement de L2/LE ont été observés dans le pays par De Mejía (2013, p. 45-47). Cette taxonomie tient compte du secteur privé et a été officialisée par le Ministère de l'Education Nationale (Bonilla \& Tejada-Sánchez, 2016). Il faut noter que le principe d'organisation de ces programmes suit le modèle d'immersion précoce (partant du modèle 
canadien) où l'exposition aux deux langues est établie en fonction d'un volume horaire déterminé.

Des écoles internationales bilingues: ce modèle se base principalement sur un curriculum international et s'appuie sur une institution ou entité d'origine étrangère. Les professeurs ainsi que les directives ou d'autres acteurs sont d'origine colombienne et étrangère. Ce qui fait accéder les élèves à des diplômes binationaux dans certains cas, par exemple, les lycées français ou des écoles allemandes, hébraïques, italiennes, japonaises, britanniques et américaines. Pour ce qui est du programme, le modèle suivi est l'immersion partielle précoce où le contact des apprenants avec la langue-cible débute à l'âge de 4 ans en moyenne. Ces institutions promeuvent une formation en espagnol-langue maternelle et une compétence renforcée de la L2 en encourageant fortement les séjours linguistiques à l'étranger et le passage d'une certification internationale de langue (le diplôme « IB » de l'organisation International Baccalauréate, ou encore d'autres diplômes de langue spécifique).

Des Ecoles nationales bilingues (anglais): ce modèle suit les caractéristiques similaires des écoles internationales pour ce qui est de la structure institutionnelle et du modèle d'immersion partielle précoce. Néanmoins, elles s'avèrent moins influencées par une présence internationale. Un trait marquant de ce type d'école est le choix de l'anglais comme L2. Ce type d'école se développe localement suivant une approche de la langue intégrée au contenu (du type EMILE $^{6}$ - d'ailleurs des recherches à ce sujet sont assez récentes en Colombie: Fandiño-Parra, Bermúdez-Jiménez \& Lugo-Vásquez, 2013). Les enseignants sont principalement des Colombiens formés en didactique des langues. Les séjours linguistiques ne sont pas promus de manière systématique, ce qui restreint le contact avec la langue cible au contexte scolaire. De Mejía (2002) souligne également que des examens internationaux de langue ont lieu pour attester le niveau de compétence en LE/L2 à la fin de l'école.

Des Ecoles ayant un programme intensif de LE (anglais): ce modèle d'école est de plus en plus commun dans les établissements du secteur public et privé. Il suit une approche d'enseignement intensif de la langue-cible, ce qui veut dire que celle-ci n'est pas intégrée dans le contenu du curriculum, mais qu'elle est enseignée comme une matière à part entière. La caractéristique principale de ces écoles est la haute intensité horaire qui permet aux élèves d'être en contact avec la langue-cible. Pour ce qui est de l'enseignement de langues dans le contexte de l'enseignement supérieur, la politique éducative de bilinguisme espagnol anglais incite donc les institutions à former des professionnels susceptibles 
de maitriser la langue à des niveaux comparables au B1 et au B2 du CECRL en anglais. Dans le cas des futurs enseignants de langue, le niveau exigé est un C1.

Comme on le voit, à travers les différent points exposés, la situation générale de l'enseignement des langues secondes et étrangères en Colombie est marquée par une forte présence de l'anglais dans les modèles éducatifs tant dans les institutions primaires, que secondaires et de l'enseignement supérieur (Cronquist \& Fiszbein, 2017; British Council, 2015).

\section{Master en Education à l'Université de los Andes}

Le Master en éducation offert par la Faculté d'éducation émerge comme une réponse aux politiques éducatives du pays qui visent, entre autres, à accroître le nombre de personnes bénéficiant de l'enseignement supérieur (OECD, 2016) ${ }^{7}$. Cette politique inclut la formation des enseignants en exercice aux niveaux de master et de doctorat ${ }^{8}$. Il propose sept parcours de spécialité: Curriculum et pédagogie; Gestion de l'éducation et politiques publiques en éducation; Éducation pour le bilinguisme; Éducation en sciences, technologie, ingénierie et mathématiques (de l'anglais, STEM); Technologies de l'éducation et de l'information et de la communication; Education pour la citoyenneté, et la petite enfance. Ce master est offert selon deux modalités d'apprentissage: présentiel et hybride (de l'anglais blended). La modalité hybride apparaît comme une opportunité d'innover du point de vue pédagogique, conceptuel et technologique, et cherche à assouplir la dynamique académique, permettant ainsi une participation plus importante et autonome des étudiants qui travaillent en parallèle ou qui habitent dans différentes régions du pays, excentrées par rapport à Bogotá, la capitale. Cette modalité a permis d'étendre la couverture au niveau national et d'améliorer les pratiques des enseignants, proviseurs ou encore professionnels impliqués dans l'éducation, ayant un impact sur différents contextes.

Le programme de Master en éducation comprend cinq cours obligatoires basés sur la recherche et trois cours facultatifs que l'étudiant définit selon ses intérêts. Dans le cursus "Education pour le bilinguisme », trois cours sont offerts: Apprentissage de langues secondes; Enseignement bilingue contextes et besoins et Processus de lecture et d'écriture en deux langues. Ces cours cherchent à examiner de manière critique les politiques, les processus et les

7 Organisation for Economic Co-operation and Development (oEcD). Organisation de Coopération et de Développement Économiques (OCDE).

8 Cette politique est connue sous le nom de « Programmes pour la formation permanente des enseignants " (en espagnol Programas de Formación Permanente de Docentes - PFPD). En ligne http://www.redacademica.edu.co/formaci\% $\mathrm{C}_{3} \% \mathrm{~B}_{3}$ n-de-docentes/formaci\% $\mathrm{C}_{3} \% \mathrm{~B}_{3}$ n/pfpds.html, consulté le 1/08/2017. 
pratiques bilingues et multilingues dans les contextes éducatifs nationaux et internationaux. Ils cherchent également à faire réfléchir les étudiants sur les contacts culturels et leur impact sur la construction d'identités dans des contextes éducatifs bilingues et multilingues.

\section{Profil des étudiants}

Les 23 étudiants du Master en Education à l'Université de los Andes qui ont participé à notre étude sont ceux qui ont choisi le parcours en Education pour le bilinguisme et le multilinguisme et se sont inscrits dans les cours de spécialité de ce parcours. Le tableau 1 ci-dessous présente l'organisation générale des enseignements, ainsi que la distribution des participants dans les cours faisant partie du parcours de spécialité:

Tableau 1. Structuration des enseignement de Master en éducation intégrés au projet - 2016-2017

\begin{tabular}{|c|c|c|c|c|}
\hline $\begin{array}{l}\text { Titre du } \\
\text { Master }\end{array}$ & $\begin{array}{l}\text { Spécialités disciplinaires } \\
\text { et de recherche }\end{array}$ & $\begin{array}{l}\text { Cours spécialité } \\
\text { éducation pour le } \\
\text { bilinguisme et le } \\
\text { multilinguisme } \\
\text { concerné par la } \\
\text { recherche }\end{array}$ & $\begin{array}{l}\text { Modalités } \\
\text { du cours }\end{array}$ & $\begin{array}{c}\text { Nombre } \\
\text { d'étudiants }\end{array}$ \\
\hline $\begin{array}{l}\text { Master en } \\
\text { éducation }\end{array}$ & $\begin{array}{l}\text { 1. Currículo y pedagogía. } \\
\text { 2. Gestión educativa y } \\
\text { políticas públicas en } \\
\text { educación. } \\
\text { 3. Educación para el } \\
\text { bilingüismo y el } \\
\text { multilingüismo. } \\
\text { 4. Educación y } \\
\text { tecnologías de } \\
\text { información y } \\
\text { comunicación (TIC). } \\
\text { 5. Educación en ciencia, } \\
\text { tecnología, ingeniería } \\
\text { y matemática (CTIM). } \\
\text { 6. Educación para la } \\
\text { ciudadanía. } \\
\text { 7. Primera infancia. }\end{array}$ & $\begin{array}{l}\text { 1. Processus de lecture } \\
\text { et d'écriture en deux } \\
\text { langues (Biliteracy). } \\
\text { 2. Apprentissage de } \\
\text { langues secondes. } \\
\text { 3. Apprentissage de } \\
\text { langues secondes. }\end{array}$ & Hybride & 11 \\
\hline
\end{tabular}

Source : création des auteures. 
Lâge des étudiants se situe entre 25 et 52 ans. 4 d'entre eux étaient des hommes et 19 étaient des femmes. Tous étaient des enseignants en exercice soit d'anglais L.E., dans la plupart de cas, soit d'espagnol LE. Les contextes dans lesquels ils travaillaient étaient des écoles publiques et privées (niveau primaire et secondaire), des instituts de langue, des universités privées, et des cours privées. Dans cette étape du projet nous analyserons les productions des 4 étudiants ayant participé au cours de processus de lecture et d'écriture en deux langues (Biliteracy).

\section{Contexte francophone: former les enseignants de français langue étrangère à la prise en compte des contextes plurilingues en France et dans le monde}

En contexte francophone, de la fin du $\mathrm{xx}^{\circ}$ au début du $\mathrm{xxI}^{\circ}$ siècle, la formation des enseignants de français langue étrangère et seconde est marquée par une prise en compte progressive du plurilinguisme individuel comme pouvant devenir l'un des éléments constitutifs des programmes de formation en didactique des langues.

\section{Le plurilinguisme : un élément constitutif des formations en didactique du français langue étrangère et seconde}

L'appellation " français, langue étrangère (FLE) » apparaît en France dès les années 60 et s'est consolidée au niveau national et international au cours des décennies 70-90. Le sigle FLE désigne un domaine distinct du domaine du Français langue seconde (FLS) qui a caractérisé dans un premier temps ce qui est associé " aux politiques de diffusion et d'enseignement de la langue française dans l'empire colonial français ", (Vigner, 1998, p. 181) et qui désigne aujourd'hui l'enseignement du français à tout individu allophone, vivant en contexte français ou, plus largement, francophone9. Placé, depuis la fin de la seconde guerre mondiale, sous la double responsabilité du ministère de l'éducation nationale (MEN) et du ministère des affaires étrangères (MAE), le FLE s'est constitué grâce d'une part à la diffusion du français à l'étranger et d'autre part, à la formation des enseignants de FLE dans le monde. À partir de 1945, le processus d'institutionnalisation du FLE 1) sera corrélé à la politique de coopération culturelle et linguistique de la France à l'étranger, 2) se positionnera

9 Il faudra attendre 2014 pour qu'ouvre une épreuve de FLs dans le Capes de lettres modernes. La préparation (facultative) de cette épreuve permet désormais aux étudiants préparant un concours de l'éducation nationale, de s'initier à la didactique du FLs et aux pédagogies interculturelles dans le cadre de leur formation d'enseignants du secondaire (Collège et Lycée). 
comme « vecteur d'une francophonie aux voix multiples» (Coste, 1999, p. 75) contribuera à la politique européenne d'ouverture interculturelle et de promotion du plurilinguisme, en s'associant aux travaux du Conseil de l'Europe, de l'élaboration d'un Niveau Seuil (1975) à celle du Cadre européen de référence pour les langues (CECRL) dans les années 200o. Mais il faudra attendre la fin des années 1980 pour que l'institutionnalisation du FLE se consolide durablement avec d'une part, la création de diplômes nationaux de français (le diplôme élémentaire et le diplôme approfondi de langue française: le DELF et le DALF), et d'autre part, la mise en place d'une filière universitaire de didactique du FLE (désormais DFLE) à caractère professionnalisant. Dès sa création, ce cursus est accueilli favorablement tant par les étudiants français (en lettres, langues et sciences du langage) qui y voient la possibilité de débouchés internationaux que par les étudiants étrangers qui viennent se diplômer pour enseigner le FLE dans leurs pays respectifs.

À ce premier public vont petit à petit s'adjoindre d'autres étudiants qui enseignent le français aux enfants et aux adultes allophones, migrants en France. En effet, à partir des années 70, une problématique s'est affirmée dans le contexte européen et singulièrement, en France et dans les ром-том: celle de l'apprentissage du français par les travailleurs immigrés (Gardin, 1976) puis celle de l'apprentissage du français langue de scolarisation (FLSCO) par les enfants nouvellement arrivés et scolarisés en France (Mendonça Diaz, 2011). Ce public enfantin va tout particulièrement poser à l'Ecole républicaine la question cruciale de son rôle face aux enfants allophones, migrants: comment y déployer les espaces de traduction, de médiation, d'interprétation, capables d'aider les enfants à faire des liens entre les langues-cultures de leurs parents et celles de la société d'accueil ? (Mesmin, 1993, p. 142).

Il faudra plusieurs décennies pour que le thème de la médiation entre plusieurs langues et systèmes culturels définisse le rôle positif de l'école et pour considérer que l'ensemble du système éducatif doit favoriser non seulement l'appropriation de compétences de communication spécifiques à une langue vivante mais l'appropriation de compétences plurilingues et pluriculturelles, dans un contexte perçu comme étant simultanément national, européen et international. Ces compétences sont définies par les auteurs de Plurilingual and Pluricultural Competence: «parler de compétence plurilingue et pluriculturelle c'est s'intéresser à la compétence à communiquer d'acteurs sociaux en mesure d'opérer dans des langues et des cultures différentes, de jouer des rôles d'intermédiaires, de médiateurs linguistiques et culturels, à même aussi de gérer et de remodeler cette compétence plurielle au cours de leur trajectoire personnelle » (Coste, Moore \& Zarate, 1997, p. 9).

La DFLE se constitue donc désormais comme promouvant une éducation à-et par- la prise en compte du plurilinguisme. Elle œuvre à la compréhension de 
compétences complexes, à acquérir tout au long de la vie et se situe résolument dans l'interdisciplinarité, entre les sciences du langage et les sciences sociales.

\section{Le Master professionnalisant en Didactique des langues de l'Université Sorbonne Paris Cité ${ }^{10}$}

L'Université Sorbonne Paris Cité (USPC) offre un master à la fois professionnalisant et axé sur la recherche, dédié à la formation d'enseignants, de formateurs et de chercheurs en DDL (didactique des langues) et DFLes (Didactique du Français langue étrangère et seconde). Ce master co-accrédité par trois établissements (Université Sorbonne nouvelle Paris $3\left(\mathrm{P}_{3}\right)$, Université Paris Descartes $\left(\mathrm{P}_{5}\right)$ et INALCO) propose une première année entièrement commune qui s'effectue en présentiel dans les 3 établissements (et également à la distance à Paris 3 ). La deuxième année se subdivise en 3 spécialités. L'objectif est de former des acteurs du monde éducatif dans le champ de l'enseignement du FLE/FLS, des langues et des cultures et de la formation plurilingue et pluri /interculturelle qui puissent, au terme du cursus, mettre en œuvre des dispositifs didactiques appropriés dans des contextes scolaires ou éducatifs variés. Sa structure en Y se justifie par l'importance à accorder, en première année, au périmètre disciplinaire complexe de la didactique des langues, notamment dans ses relations avec les disciplines connexes (linguistique, sociolinguistique, anthropologie culturelle, littérature) et à permettre de se spécialiser en deuxième année en fonction de contextes et de publics distincts (FLE/FLS) ou d'objectifs professionnels plus spécifiques (ingénierie de la formation). La polyvalence disciplinaire et professionnelle de la formation permet de répondre aux nouvelles dynamiques sociétales et scientifiques de la didactique des langues. Elle fournit aux étudiants les conditions d'une bonne adaptation au marché des langues en pleine évolution ainsi qu'une meilleure compréhension des politiques linguistiques et éducatives qui les façonnent. La formation marquée par la pluridisciplinarité, la polyvalence, la réflexivité et le travail collaboratif permet de répondre à un marché de l'emploi très dynamique en France et à l'international. Elle est tendue vers des objectifs scientifiques et professionnels qui sont complémentaires dans la professionnalisation des étudiants. La formation permet aux étudiants de construire des compétences théoriques et pratiques en didactique du FLE ou FLS pour exercer le métier d'enseignant de français et de langue(s), en présentiel ou à distance, des activités de conseil pédagogique et d'encadrement de formateurs et/ou pour organiser et expertiser des formations dans le domaine,

10 Cette partie reprend les principaux éléments du rapport rédigé en 2017 par Valérie Spaëth, directrice du Master DDL à Paris 3, à la demande du Haut conseil de lévaluation de la recherche et de l'enseignement supérieur (HCERES). 
en France ou hors de France, dans divers contextes éducatifs monolingues ou plurilingues et pluriculturels. Il s'agit donc d'acquérir à la fois des compétences générales en didactique du français et des langues, fondées sur des savoirs disciplinaires en littérature, en anthropologie culturelle, en sciences du langage et des compétences plus spécifiques, qu'elles soient relatives à des langues données ou qu'elles soient transversales (communication interculturelle, prise en charge de l'analphabétisme et l'illettrisme, analyse de situations éducatives complexes, ingénierie des formations dans le domaine du français et des langues). Les stages (courts en Master 1 - M1 et longs en Master 2 - M2) permettent aux étudiants de se positionner progressivement comme des professionnels dans le champ. Les débouchés sont variés:

- Dans l'enseignement: le secteur de la recherche publique et de l'enseignement supérieur (avec poursuite d'études en doctorat) ; le secteur de l'enseignement du français langue étrangère, au niveau national et international (soit dans le cadre d'un détachement par le MAE pour les titulaires de l'éducation nationale, soit dans le cadre de recrutements locaux à l'étranger ou en France dans des organismes de formation ou dans des écoles de langues). Le master DDL permet aussi à des titulaires des concours de l'éducation nationale (professeurs des écoles et enseignants du secondaire) de se spécialiser dans l'enseignement à des enfants étrangers nouvellement arrivés.

- Dans d'autres secteurs tels que les métiers du secteur de la formation (conseil pédagogique, encadrement et formation de formateurs) ; métiers du secteur de l'édition papier et/ou numérique ; métiers du secteur des relations internationales et services culturels (Coopération linguistique et éducative auprès du Ministère des Affaires Étrangères); métiers de l'administration territoriale (Chargé de mission aux politiques de la ville, de l'intégration).

De manière générale, l'employabilité des étudiants sortis de master DDL est satisfaisante comme l'indiquent les enquêtes d'insertion professionnelle des établissements respectifs (environ $2 / 3$ des étudiants ont un emploi 18 mois après leur diplôme et les $4 / 5$ après 30 mois). Les étudiants trouvent assez facilement de l'emploi, en France comme à l'étranger, mais le salaire plafonne vite et les possibilités de carrière sont très inégales. En effet, les étudiants étrangers employés dans leurs pays respectifs ont en moyenne des postes et des possibilités de carrière plus favorables que pour les Français, employés en France ou à l'étranger, qui à moins d'être fonctionnaires (de l' l'Education nationale généralement) ont des emplois plus précaires. Le master DDL jouit d'un bon rayonnement international. En effet, pour l'ensemble du diplôme, presque la 
moitié des étudiants recrutés chaque année sont de nationalité extrêmement diversifiées (roumaine, grecque, polonaise, russe, chinoise, indonésienne, algérienne, marocaine, brésilienne, colombienne, etc.) Ces étudiants sont souvent boursiers de leur gouvernement. La forte présence des étrangers dans le diplôme constitue une richesse évidente pour la formation.

La professionnalisation par la recherche et l'approche réflexive en didactique des langues est valorisée. En effet, la formation propose dès le M1 une initiation aux méthodes de recherche en DDL qui se poursuit en M2 dans chaque spécialité. Les étudiants peuvent envisager une orientation vers les métiers de la recherche et de l'enseignement supérieur avec une poursuite d'études en doctorat - principalement de Didactique des langues et des cultures à Paris 3. L'approche réflexive est développée dès le $\mathrm{M} 1$ et tout au long de la formation. Elle favorise la construction de l'ensemble des compétences didactiques visées au sein d'ateliers pédagogiques de stages d'enseignement tuteurés et/ou en responsabilité. La formation dans son ensemble est fortement articulée à la recherche grâce aux 3 équipes d'accueil (DILTEC ${ }^{11}$ EA $2288-\mathrm{P}_{3}$; EDA ${ }^{12}$ EA $4071-\mathrm{P}_{5}$ et PLIDAM $^{13}$ EA 4514 - INALCO) qui y sont directement impliquées d'une part, par le nombre d'enseignants chercheurs qui assurent séminaires et directions de mémoires et d'autre part, par les projets impliquant les masterants. À Paris 3, la continuité entre le département de FLE, l'équipe DiLTEC et le doctorat de Didactique des langues et des cultures, favorise une bonne articulation entre recherche et formation et une grande cohérence et fluidité dans le parcours de formation.

\section{Profil des étudiants de Master 1 et 2 en didactique des langues et des cultures}

Notre recherche-action intitulée Approche comparée des dispositifs de formations des formateurs à la dimension interculturelle explicite dans l'enseignement des langues étrangères et secondes est co-pilotée par quatre enseignantes-chercheures (les trois auteures de cette contribution ainsi qu' Anne-Marie Truscott de Mejía), ayant à cœur de susciter au sein même de leurs masters, un dialogue international et interdisciplinaire en sciences de l'éducation, acquisition des langues et en didactique des langues et des cultures. Nos finalités sont d'élaborer un certain nombre de réponses et de préconisations autour des nécessités suivantes:

11 Didactique des langues, des textes et des cultures: http://www.univ-paris3.fr/diltec-didactiquedes-langues-des-textes-et-des-cultures-ea-2288-3451.kjsp?RH=1179925961149.

12 Éducation, Discours, Apprentissages: http://eda.recherche.parisdescartes.fr/.

13 Pluralité des Langues et des Identités en Didactique: Acquisitions, Médiations: http:// www.inalco.fr/recherche/equipes-recherche/pluralite-langues- identites-didactique-acquisitionmediations-plidam-4. 
- Prendre en compte la diversité des contextes dans lesquels s'effectuent les expériences bilingues, multilingues, plurilingues et interculturelles des apprenants ;

- Promouvoir des processus d'empowerment chez les enseignants en faisant en sorte que la diversité de leurs parcours individuels et histoires collectives deviennent des vecteurs de formation;

- Elaborer des ressources cognitives, méthodologiques, éthiques, ensemble et les partager.

Les 30 étudiants de Paris 3 ayant participé à cette recherche se répartissaient dans 2 des spécialités proposées en deuxième année du Master en didactique du FLE/FLS et des langues. Nous indiquons dans la colonne de droite, les cours concernés par notre recherche-action.

Les étudiants en Master 1 et 2 ayant participé à cette recherche et suivant des enseignements en présence ou à distance, étaient 26 femmes et 4 hommes, entre 20 et 51 ans. Ils travaillaient dans des associations, des instituts de langue, des écoles publiques ou privées (de niveaux primaire et secondaire), à l'université, ou dispensaient des cours particuliers. Dans la partie du projet que nous divulguons ici, seront analysées les productions écrites et multimodales des 7 étudiants du cours de didactique de l'écrit réflexif en modalité présentielle.

\section{Une épistémologie des savoirs pédagogiques, cognitifs, pratiques et expérientiels}

La diversité de nos domaines de recherche et approches épistémologiques illustre notre désir d'avancer dans une perspective pluridisciplinaire. Adoptant une posture éthique dans la compréhension d'un certain nombre de préoccupations sociétales, nous explorons les effets de l'enseignement-apprentissage des langues du point de vue d'auteurs tels que Bourdieu \& Passeron (1977) ou Jarvis (2006). En effet, si l'éducation est obligatoire (par exemple, en France), elle n'est pas accessible à tous (par exemple en Colombie), elle peut être perçue de façon positive ou négative car pour certains enfants, aller à l'école ressemble à une punition, là où pour d'autres, la punition est de ne pas d'aller à l'école...Enfin, les clivages entre communautés majoritaires et communautés minoritaires loin de s'estomper, semblent se creuser davantage (Harris, 2011; Unesco, 2003) ${ }^{14}$.

Si nous avons choisi pour notre recherche, de privilégier une approche empirico-inductive-qualitative et critique (cf. nos développements ci-dessous), c'est 


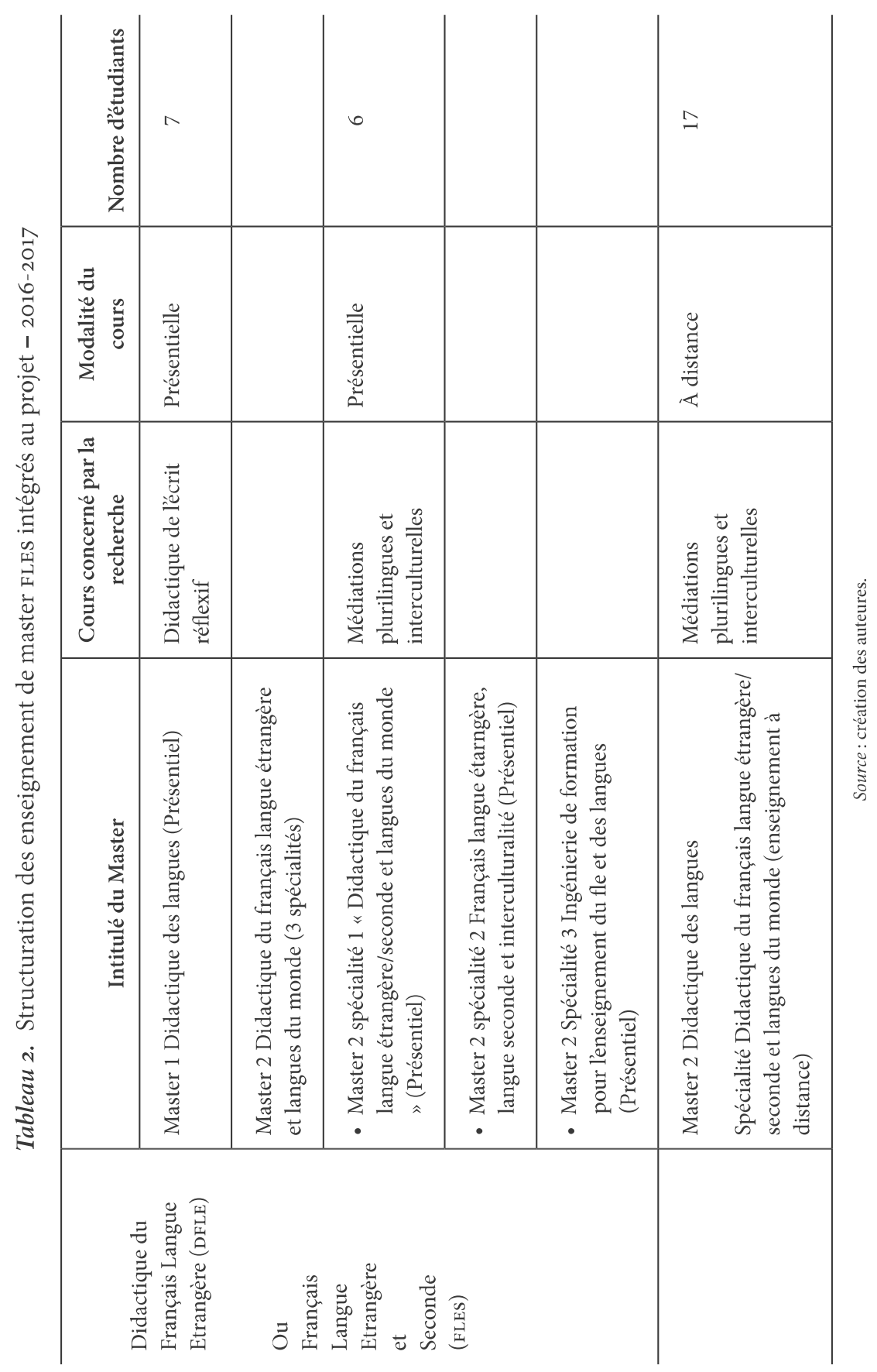


donc dans l'intention non seulement de comprendre ces inégalités culturelles, mais aussi de produire des connaissances permettant à nos étudiants (futurs enseignants) d'intervenir sur ces inégalités et sur les effets qu'elles produisent sur le devenir de nos sociétés, tant en France qu'en Colombie.

\section{Une approche empirico-inductive-qualitative}

C'est ainsi que tant notre projet scientifique que notre projet de formation à- et par- la recherche reposent sur une méthode empirico-inductive qualitative qui propose:

« une compréhension de phénomènes individuels et sociaux observés sur leurs terrains, en prenant prioritairement en compte les significations qu'ils ont pour leurs acteurs eux-mêmes et donc en vivant ces phénomènes aux côtés des acteurs, comme un acteur parmi d'autres mais selon des procédures méthodiques qui garantissent la significativité des situations observées et comparées et qui exploitent consciemment les relations intersubjectives entretenues au sein du groupe, notamment celles où le chercheur est impliqué. Ce sont donc les phénomènes observés qui induisent l'interprétation: il n'y a pas d'hypothèse de départ (au sens d'une " réponse prédéterminée " à valider ou invalider) mais un questionnement auquel la recherche permet d'apporter des éléments de réponse (on parle en anglais de data driven research, « recherche pilotée par les données ») »(Blanchet \& Chardenet, 2011, p. 15-16).

A l'instar de Blanchet et Chardenet, nous mettons en œuvre (et formons nos étudiants à mettre en œuvre dans leurs recherches en master 2) les quatre principes épistémologiques caractéristiques de ce type d'approche à savoir:

- Le principe d'humanisme: en effet nous considérons les humains, individus et/ou groupes comme des sujets autonomes, dignes, respectables, riches de toute leur singularité,

- Le principe d'intersubjectivité: dans le sens où c'est dans et par les interactions entre les sujets et les significations qu'ils y attribuent, chercheur compris, que se développent et peuvent être compris les phénomènes humains et sociaux,

- Le principe d'endo-référentialité: nous cherchons à comprendre les sujets à partir de leur propre système de référence, de valeurs, de significations,

- Le principe d'hétérogénéité: les phénomènes humains et sociaux, les humains, sont avant tout marqués par leur extrême hétérogénéité, 
complexe, qui interdit de les réduire à des règles universelles et qui permettent de proposer des connaissances profondément situées, en dégageant éventuellement des tendances partielles mais jamais des prédictions absolues op. cit.

\section{La pensée critique}

Explorée comme le proposent Weil \& Kincheloe (2004), la pensée critique permet la problématisation des réalités éducatives basées sur le raisonnement, à travers des logiques diverses ou visions plurielles de compréhension du monde. Nous considérons que cette approche nous aide à trouver des possibilités variées de voir et d'appréhender nos systèmes éducatifs. En effet, elle donne aux enseignants et aux apprenants les outils pour appréhender les interactions entre textes, idées objets physiques, circonstances sociales ou processus pédagogiques préalablement ignorés. Aborder l'enseignement-apprentissage des langues via l'analyse des questions associées à notre positionnement dans le monde ou dans le tissu de ses réalités influence nos façons de nous percevoir et de percevoir (Weil \& Kincheloe, 2004).

\section{La pédagogie critique}

Adoptant le point de vue d'auteurs comme Paulo Freire (2006), Henry Giroux (2004) ou Peter McLaren (1995) nous étudions la corrélation entre l'école, les programmes d'études et les faits sociaux. Nous considérons que professeurs et étudiants peuvent réfléchir ensemble sur les contenus enseignés et/ou appris; sur comment le processus d'enseignement-apprentissage se met en place ou sur les effets des modèles d'enseignement-apprentissage qui reproduisent des formes d'exclusion sociale. La pédagogie critique nous aide également à problématiser et à nourrir les réflexions liées à la co-construction des curriculums ou des propositions didactiques interculturelles. En ce qui nous concerne, il s'agit d'évaluer, de comprendre et d'associer les cultures éducatives, Beacco Chiss, Cicurel \& Véronique $(2005)^{15}$ que caractérisent nos programmes de formation

des enseignants de master en éducation et de master en didactique des langues et des cultures, ainsi que les pratiques d'enseignement-apprentissage.

15 Les cultures éducatives renvoient à l'idée que les activités éducatives et les traditions d'apprentissage conditionnent en partie enseignants et apprenants. 


\section{La théorisation ancrée ou Grounded theory}

Notre recherche s'appuie sur une méthode de construction théorique à base empirique connue sous le nom de théorisation ancrée (TA) ou Grounded Theory. Ici, la théorie n'est pas formulée à partir d'hypothèses préalablement conçues mais découle au contraire des données analysées (Glasser \& Strauss, 1967; Charmaz, 2006). La TA s'inscrit donc dans la recherche qualitative dans un cadre inductif et suivant une approche constructiviste. La TA est fréquemment associée à l'enquête de terrain ethnographique. Cependant, il convient de différencier la TA de cette dernière car le chercheur qui applique une TA décrit des phénomènes psychosociaux de base dans le but de comprendre ou d'expliquer, par le biais d'une théorie ou d'un modèle, comment les sujets se servent des interactions pour construire des réalités contextualisées.

Le procédé pour appliquer la TA consiste à recueillir et analyser les données en amont. Une fois les données obtenues et organisées, il faut les catégoriser à partir des éléments clés dérivés de la littérature pertinente dans un processus de codage. Dans notre cas, elles sont encodées sous plusieurs formats (audiovisuel, texte, questionnaire). Des procédés différents se trouvent dans la littérature. Strauss \& Corbin (1990) font référence à trois étapes dans le codage des données: le codage ouvert, le codage axial et le codage sélectif. D’autres auteurs comme Charmaz (2006) ont parti de cette organisation du codage pour en proposer des processus de codage contextualisés et rigoureux. Dans le codage ouvert, les données brutes sont observées et triées selon les objectifs des chercheurs. Dans l'étape suivante, le codage axial, des éléments clés, souvent appelés des codes, sont attribués à des échantillons de données pour regrouper des thématiques pertinentes qui se relient aux questions de recherche de l'étude. Enfin, la dernière étape, le codage sélectif, renvoie à des catégorisations. Ces catégories sont à la base de la configuration d'une théorie, d'un modèle, ou de l'intégration des constats à une théorie existante qui servent à expliquer le phénomène en question.

\section{Notions clés}

Viser une formation explicite aux dimensions interculturelles nécessite l'adoption des notions transversales et connexes à nos domaines et intérêts de recherche. Pour ce qui est des participants à cette étude, ce sont des étudiants de master engagés dans l'enseignement des langues. Notre proposition s'appuie sur une co-construction des dimensions altéritaires, partant de la compréhension du rôle du langage dans la relation à l'autre, dans les différentes lectures du monde, et les réflexions sur soi-même et autrui. 


\section{Dimensions interculturelles}

La nécessité d'intégrer les dimensions interculturelles ${ }^{16}$ dans l'enseignement des langues est largement débattue, depuis plusieurs décennies. Les finalités des recherches et des enseignements axés sur l'interculturel se sont peu à peu transformées passant d'une première étape: rendre possible la communication avec des locuteurs d'une langue cible, notamment dans un autre pays (Blanchet \& Martinez 2010), à une seconde étape: accepter l'hétérogénéité inhérente à nos sociétés dans lesquelles se produisent des rencontres, des interactions, des relations entre locuteurs qui originaires (ou pas) du même pays, ville ou famille et possédant des identités linguistiques et culturelles multiples (Abdallah-Pretceille, 2006). Ces locuteurs peuvent à la fois appartenir au groupe tout en revendiquant leur individualité ou idio-culture (Gamboa-Diaz, 2014), entendue comme l'ensemble des paramètres de comportement individuels qui changent et s'actualisent en fonction des circonstances auxquelles les personnes sont exposées, De ce fait, les résultats des interactions (contacts, rencontres de nature verbale ou non verbale avec autrui) sont imprévisibles, d'un individu à l'autre. Sur le plan des échanges, les paramètres idio-culturels, déterminent également les intentions communicationnelles et le type de relation que les participants souhaitent engager (Molinié, 1993).

Pour Blanchet \& Martinez (2010), la notion d'interculturalité prend désormais en compte l'inévitable métissage engendré par tout contact culturel. Pour Byram et al. (2002), la dimension interculturelle concerne autant les appartenances culturelles que linguistiques et de ce fait, les relations interindividuelles sont promotrices de l'interculturalité, sans que cela implique une quelconque appartenance prédéfinie pour les apprenants-locuteurs. Pour Mignolo, Walsh y García Linera (2006), Rojas Curieux et Barona Becerra (2007) ou Rojas (2011), l'interculturalité est associée à la défense des droits des communautés dites " minoritaires ", à la reconnaissance équitable, juste de la diversité culturelle et linguistique, à la production de connaissances et d' interactions entre les membres des communautés «minoritaires et majoritaires». Pour nous, l'interculturalité est la capacité d'explorer, d'analyser les similitudes, les différences ou les éléments inattendus d'une autre culture (individuelle [Idio-culture] ou collective) dans le but de développer (autant que possible) des stratégies d'ajustement situationnel, communicationnel, relationnel, etc. Il s'agit d'adopter une

16 Adaptée de la proposition initiale de dimension interculturelle de Byram et al., 2002 nous considérons la multidirectionnalité de ce concept et le présentons ici comme un macro-concept à partir duquel peuvent être englobés la notion d'interculturalité, le paradigme interculturel, léducation interculturelle, la perspective interculturelle, la communication interculturelle et la compétence interculturelle. 
perspective interactionnelle critique, de comprendre et d'associer la diversité à des personnes connues et inconnues, à des situations courantes et inattendues ainsi qu'à des contextes multiples (proches et lointains) (Gamboa-Diaz, 2016).

Ainsi, nous considérons pour notre part, que l'interculturalité repose sur les capacités qu'ont les individus (multi-appartenants) de nouer des relations dialogiques, dans l'intention réciproque de s'engager dans une dynamique relationnelle intersubjective, et prenant en compte à la fois la singularité de cette dynamique ${ }^{17}$ et un certain nombre de facteurs contextuels, en synchronie (ici et maintenant) et en diachronie (historicité).

Pour ce qui est des contextes socio-éducatifs colombien et français, les rencontres, les interactions langues et cultures présentes à l'intérieur et en dehors de la salle de classe déterminent les dimensions interculturelles. Ces dernières sont une partie intégrante des cursus de formation et doivent être incluses de manière explicite dans les curricula. Les individus (consciemment ou pas porteurs de culture) sont en contact dans des situations de migration, de voyage ou toute autre forme de déplacement ainsi que dans les situations d'enseignement-apprentissage. Enseigner et apprendre la langue de l'Autre ce n'est pas seulement être confronté à sa culture (Kramsch, 2013) mais, de façon plus dynamique et plus complexe s'ouvrir à son répertoire culturel singulier et, par conséquent, à la possibilité de transformations des identités des participants (professeurs et apprenants) dans des processus éducatifs, communicationnels et relationnels (Molinié, 1993).

\section{Littératie}

Nous présentons ici une caractérisation de la notion de littératie adoptée dans les deux contextes de formation d'enseignants de cette étude en didactique des langues étrangères/secondes. La notion de littératie concerne non seulement l'éducation en général, mais aussi le domaine de la linguistique appliquée. Elle fait l'objet de recherches en psycholinguistique de l'individu (en relation avec le développement cognitif de celui-ci), ainsi qu'en anthropologie et en sociologie (en relation avec la construction du sujet et de son rôle social). Cette notion concerne particulièrement la formation des enseignants de langue car, dans sa définition la plus ample, elle renvoie au rôle du langage dans la construction des savoirs et dans la relation du sujet (apprenant, citoyen) avec le monde.

Lorsqu'on réfléchit à la littératie, il est fréquent de se situer dans une vision instrumentale. Les processus les plus fréquemment rattachés à ce concept sont ceux de la lecture et de l'écriture. Dans sa revue des études des littératies (literacy 
studies), Williams (2006) fait référence à deux perspectives pour aborder cette notion: une perspective restreinte (narrow) et une perspective ample (broad). Dans la perspective restreinte, l'on retrouve la conception de ces habiletés à des fins de 'décodage' des contenus et de construction des savoirs. Williams signale par ailleurs, une traditionnelle séparation de la lecture et de l'écriture dans les contextes éducatifs. Cette séparation renvoie à la conception de la lecture comme un processus plus 'élémentaire' et nécessairement préalable à l'écriture (Venezky, 1990, cité par Williams, 2006). Dans cette optique, on place la lecture et l'écriture au centre des préoccupations du système scolaire et par conséquent, l'approche pédagogique de la littératie devient un enjeu important dans le domaine didactique. À ce sujet, Chiss (2004) et Goody (1968) signalent la nécessité de s'interroger sur les moyens (techniques, pédagogiques, méthodologiques) de l'école dans l'appropriation des savoirs dans « une perspective communicationnelle et culturellement marquée des usages [de ces littératies] ». (Moore et Molinié, 2012, p. 5).

La dimension communicative, signalée par Moore et Molinié (2012) et Williams (2006) est au centre d'une nouvelle conception de la littératie. En ce qui concerne la didactique de langues secondes et étrangères, les littératies ont cessé de renvoyer à des activités de décodage futile. Au contraire, elles sont au service de l'apprenant et de l'enseignant pour s'approprier une nouvelle façon de communiquer et de construire les identités (Barkhuizen, 2017) et perceptions du monde. Cette perspective ample proposée par Williams (2006) et adoptée dans notre démarche, se situe dans le sillage des nouvelles études de littératies (new literacies studies) développées dans les trois dernières décennies. Ici le sujet apprenant est confronté à des réalités complexes présentées dans des modalités diverses: à l'oral, à l'écrit, dans les medias traditionnels et numériques et dans différentes formats. Les mécanismes dont se sert l'apprenant pour comprendre et construire une posture critique vis-à-vis de ces réalités sont au cœur des nouvelles littératies. Pour ce qui est de la formation de formateurs, Knobel \& Kalman (2016) parlent d’un « virage digital » dans les nouvelles études de littératies (en anglais digital turn). Ceci fait référence à l'importance du rôle des technologies dans la conception d'une pratique pédagogique située et prête à comprendre les défis d'un public qui se transforme rapidement. Dès lors, l'enseignant doit " anticiper au-delà du moment présent et envisager comment éduquer de la meilleure manière aujourd'hui pour promouvoir les capacités des apprenants, construire des significations et communiquer dans un futur prévisible» (Lamkshear \& Knobel, 2014, p. 97, cité par Knobel \& Kalman, 2016 $)^{18}$.

18 "Anticipating beyond the present and envisaging how best to educate now in order to enhance learner's capacities for effective meaning making and communication in a foreseeable future." (Lamkshear \& Knobel 2014, 97 cité par Knobel \& Kalman 2016, p. 5). Traduction de l'auteur. 
Enfin, comme le signalent Moore \& Molinié (2012), cette extension du domaine de la littératie invite les didacticiens à un « élargissement du champ de leur réflexion sur les pratiques sociales du lire-écrire » (p. 5). Cet élargissement fait recours à toutes les dimensions de la connaissance mais aussi aux technologies qui ont décidément transformé la manière dont on perçoit le monde et construit les savoirs et savoir-faire. Dans cet objectif, les deux auteures proposent d'entendre ces pratiques de lecture et d'écriture comme un continuum qui s'étend entre différents facteurs, modalités, formes et langues.

Sur les deux terrains explorés dans cette étude (en France et en Colombie), les nouvelles littératies ont été centrales. En fait, elles ont constitué un tronc commun pour la construction d'expériences pédagogiques fondées sur les échanges interculturels à l'intérieur- et entre- les deux contextes. Enfin, nos propositions suivent les réflexions de Moore et Molinié affirmant:

Notre projet (...) s'enrichit du fait que cette notion élastique, pour l'essentiel emprunté aux travaux anglo-saxons, subit des passages transcontinentaux et d'une langue à l'autre, véhiculant d'une rive à l'autre des emplois pluriels: nouvelles littératies, multilittératies, littératies plurilingues, plurilittératies, etc. (Moore \& Molinié, 2012, p. 7).

\section{Une articulation nécessaire entre réflexivité, processus d'autoformation et de recherche}

Pour nous, l'articulation entre réflexivité et formation dans le domaine spécifique des langues, des cultures et de l'interculturel, repose sur l'hypothèse générale de cette « réflexivité radicale » qui, pour Taylor (1989) caractérise l'homme moderne, poussant celui-ci à une réflexion permanente sur ses conduites, objectifs de vie et moyens pour les réaliser. Nous évoluons dans un contexte historique qualifié de post-modernité et/ou d'hyper-modernité (Aubert, 2004) dans lequel des individus sont « de plus en plus libérés à la fois des contraintes culturelles imposées par la religion, la tradition et la moralité conventionnelle, et de contraintes structurelles, telles que la classe, le statut, le genre et la famille nucléaire. Dans la mesure où les formes traditionnelles perdent leur force contraignante, les individus peuvent, en principe, réfléchir librement sur la vie qu'ils veulent mener et la façonner comme ils veulent. L'identité personnelle devient une opération réflexive ». (Vandenberghe, 2006, p. 977). Conscient de cela, nous avons, en tant qu'enseignants-chercheurs-formateurs, des missions et des responsabilités particulières: répondre au besoin d'accroissement des ressources langagières dans l'expression de l'identité personnelle et professionnelle de nos étudiants; favoriser le développement de leurs pratiques réflexives à- et 
par- la recherche; augmenter leurs possibilités (individuelles et collectives) d'articuler toujours plus dans le langage savoirs expérientiels et savoirs théoriques. C'est ainsi qu'en master nous proposons des dispositifs de formation qui associent les verbalisations (orales et écrites) à d'autres modes d'expression (dessin, collages, mises en espace théâtral, etc) afin que puisse s'établir un continuum entre moments de constitution du répertoire didactique de chacun, mises en mouvement d'un imaginaire etprocessus d'élaboration de savoirs. Ces éléments confirment l'intérêt d'une approche qualitative, compréhensive, réflexive et interprétative du plurilinguisme qui:

- S'intéresse autant aux pratiques qu'aux techniques, met au travail les sujets à partir de leur expérience située, en mobilisant à la fois leur réflexivité et leur imaginaire (Auger, Dervin et Suomela-Salmi, 2009), dans un cadre critique de déconstruction de représentations sociales qui maintiennent les étudiants/futurs enseignants dans des formes (discrètes mais efficientes) d'insécurité linguistique et cognitive ;

- Met en résonance les expériences individuelles qui deviennent un puissant opérateur de compréhension du social;

- S'appuie sur la pluralité « des rythmes, des mémoires, des lectures, des intelligences, des types de compréhension; [de l'] existence de quatre grammaires de la langue: deux grammaires de la langue écrite (écriture et lecture), deux grammaires de la langue orale (parole et écoute); [de la ] relation ininterrompue de l'action et de la perception qui, l'une et l'autre, élaborent l'histoire de notre couplage à l'environnement ; accepter d'admettre que notre perception crée notre vision du monde ; accepter de voir dans l'erreur un écart à la norme demandée mais aussi un indice du processus suivi, de la stratégie adoptée, de la ressource utilisée » (Trocmé-Fabre, 1987, p. 245).

La socialisation des expériences est un grand principe qui doit permettre aux futurs enseignants de développer leur autonomie et leur capacité à créer à leur tour, des dispositifs originaux et contextualisés qu'ils institueront selon des procédures négociées avec leurs futurs apprenants. Former des enseignants consiste donc à développer leurs capacités à penser leur action jusqu'à en faire un objet de recherche.

\section{Réflexivité, formation et action}

Nous situant dans l'orientation dégagée par les travaux de Perrenoud (1999), nous considérons l'action comme étant toujours non seulement située mais 
insérée dans un système. L'action procède d'une intention et s'inscrit dans un système de rapports sociaux et d'actions collectives. Le praticien réflexif est donc un professionnel sachant réfléchir au quoi faire ? Quand ? Comment faire? Il sait tenir compte de son environnement institutionnel et de ses publics. C'est pourquoi, acquérir une posture réflexive équivaut à acquérir une capacité à réfléchir sur sa propre action et à en tirer des savoirs qui permettront au professionnel de modifier ses attitudes et comportements dans une situation analogue. Trois principes guident donc notre travail dans le domaine de la formation des enseignants.

Premier principe, la formation s'appuie sur l'action située des individus «se formant " pour faire de celle-ci un objet de parole et d'apprentissage. Par conséquent, un premier niveau de réflexivité désigne la réflexion qui porte sur la pratique, qui a lieu pendant ou après l'action et a pour visée d'en tirer des enseignements (pour ensuite valider ou modifier la pratique). Ce «primat de l'action " nous encourage à renoncer à toute illusion applicationniste dans notre domaine et à faire jouer à l'écriture un rôle majeur dans la mise en œuvre des processus réflexifs associés à l'action (par exemple, dans des portfolios socioconstructivistes). Activant le lien dynamique entre langage et pensée, entre pratique langagière située et activité cognitive, l'écriture peut en effet favoriser diverses opérations réflexives (en milieu scolaire comme en formation professionnelle).

Second principe, l'on peut produire de la connaissance à partir de l'expérience vécue. C'est ainsi que dès la licence, les étudiants de FLE sont formés à un premier niveau de réflexivité grâce au cours intitulé " apprentissage réflexif d'une langue inconnue » (ARLI) et à l'outil « Journal de bord des apprentissages » (Cadet, 2007). Ce dispositif développe un type de réflexivité (la métacognition) qui consiste en « retours du sujet sur ses opérations cognitives » et peut « aboutir à un jugement (...) sur la qualité des activités mentales en question ou de leur produit et éventuellement à une décision de modifier l'activité cognitive, son produit ou même la situation qui l'a suscité » (Noël, 1991, p. 19). Mais cela ne suffit pas à former des praticiens capables de faire face à l'ensemble des situations réflexives existantes ou requises aujourd'hui par les processus d'enseignement et les processus d'apprentissage.

Le troisième principe est donc très important: la formation professionnalisante n'est pas seulement vue comme mise en forme de matériaux pré-existants mais comme re-configuration et transformation de ces matériaux.

Ayant acquis ces trois principes, le but de l'enseignant dans sa classe ne sera pas seulement l'accroissement du stock de connaissances linguistiques et culturelles de l'élève (ou de l'étudiant) mais 1) l'exploitation d'un « déjà là », constitué par le répertoire plurilingue et pluriculturel de cet élève, et 2) la re-configuration et transformation de ces matériaux. 


\section{Méthodologie : la recherche action}

La pratique éducative nécessite d'une réflexion permanente si elle se veut actualisée, consciente et pertinente. C'est dans cet esprit de remise en question de l'objectif de l'éducation que la recherche action s'instaure comme un champ grandissant de la recherche éducative et des sciences sociales. La recherche action est conçue comme un processus systématique qui vise à étudier de manière directe et constante des phénomènes issus de la pratique professionnelle tout en cherchant une meilleure compréhension de ceux-ci (Burns, 2015).

En tant que stratégie de recherche, le but principal de la recherche-action est de «fournir un cadre aux recherches qualitatives effectuées par les enseignants et les chercheurs en situations complexes de classe» (Catroux, 2002, p. 5). Dans le cas de cette étude, cette stratégie a été adoptée afin d'atteindre deux objectifs principaux. D'un côté, pour enrichir la formation des enseignants des L2/LE et fournir un espace d'autoréflexion sur leurs propres idées, préconceptions, propositions d'action et d'autoévaluation notamment concernant les dimensions interculturelles. D'un autre côté, pour nourrir notre propre exercice de formatrices, de formateurs et d'enseignantes-chercheuses.

Partant d'une nature cyclique, la recherche action a été notamment représentée par une figure en spirale. Celle-ci propose de diviser les étapes de la réflexion afin de guider le chercheur à identifier, d'une part, les sources des phénomènes potentiellement problématiques ou susceptibles d'être explorés et, d'autre part, les routes et les mécanismes d'action qui sont les plus appropriées selon la situation. Le modèle présenté ici, adapté de celui de McNiff (2013), est utilisé dans l'esprit de fournir aux étudiants et aux chercheuses les étapes suivantes de formation et de recueil d'information:

1. Constat et analyse d'un phénomène (Reflect) ; situation problématique ou d'intérêt pour les enseignants-chercheurs en devenir ou confirmés dans le cadre de sa pratique éducative quotidienne.

2. L'exploration de ce phénomène dans le but d'observer ses causes ou ses associations et de tracer une action qui vise à le modifier ou l'améliorer (Plan).

3. La mise en œuvre de cette action dans le cadre de la pratique éducative réelle $(A c t)$.

4. L'évaluation de cette action, ce qui implique la validation et le questionnement (Observe). 


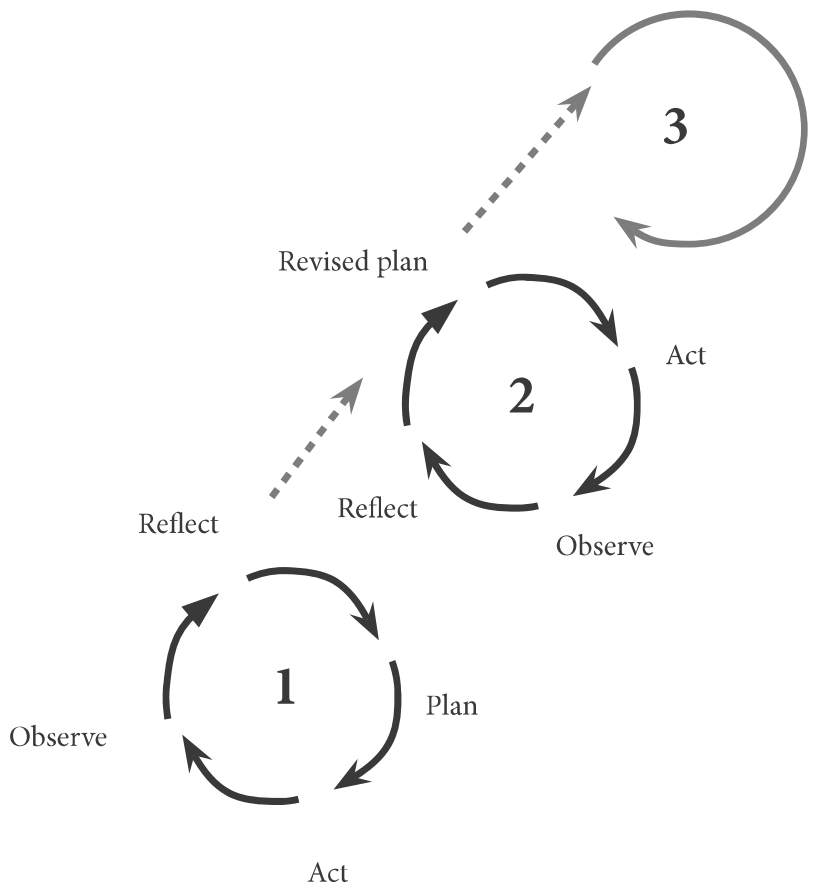

Figure 1. Cycles de la recherche-action en spirale

Source : création des auteures à partir de McNiff, 2013.

\section{Présentation du dispositif de formation des (futurs) enseignants de langue}

Dans ce projet nous adoptons la recherche action dont les visées conceptuelles et praxéologiques sont, de notre point de vue, étroitement liées à la théorisation ancrée. En effet, dans l'objectif de construire une proposition de formation explicite aux dimensions interculturelles, nous ne partons de préconisations toutes faites mais des données obtenues dans nos deux contextes. Ce rôle primordial du corpus recueilli et observé est à la base de ces deux approches. D’après Dick (2007), la recherche action s'appuie sur les méthodes et procédés exposés par la théorisation ancrée, tel que les codages, pour rendre compte d'une analyse rigoureuse des informations produites tout au long du processus et non seulement dans l'étape finale du cycle, celle de la réflexion.

Nous appuyant donc sur la recherche action et la théorisation ancrée, notre projet interuniversitaire tente de mettre en regard deux programmes de master, de façon à expliciter les approches qui les sous-tendent, dans le but de construire une culture éducative commune autour d'éléments centraux (tels 
que l'interculturalité et la réflexivité) en réponse à certaines questions sociales vives de ce début de $\mathrm{XXI}^{\circ}$ siècle, telles que la prise en compte des processus de métissages linguistiques et culturels, mais également, le déséquilibre entre les classes sociales, les situations de violence physique, attentats, de stress psychologique, ou encore les contextes de sortie du conflit armé.

Les productions multimodales de nos 7 enseignants-formateurs de français langue étrangère, et seconde, en France, ainsi que nos 4 enseignants-formateurs d'anglais langue étrangère ou seconde en Colombie, nous ont permis d'accéder aux données ci-dessous listées.

1. Les réponses aux questionnaires en ligne.

Les étudiantes ont rempli deux questionnaires, en début et à la fin du semestre. Le but de cette activité était de découvrir le parcours personnel, académique et professionnel de nos participants, ainsi que leurs perceptions et / ou connaissances préalables, associées à l'interculturalité.

2. Les productions écrites et audiovisuelles associées au parcours personnel. Des lettres et des vidéos ont été échangées à travers la plate-forme Moodle. Les étudiants en France écrivaient en français et les étudiants en Colombie en espagnol, dans le but d'appliquer le principe d'intercompréhension $^{19}$. Les écrits et les vidéos comportaient des messages socio-biographiques esquissant a) une courte présentation personnelle et b) une autobiographie de lecteur et de scripteur.

3. Les productions écrites associées au parcours académique et professionnel. À la suite de la lecture des textes proposés dans le cadre du cours, les étudiants ont été guidés vers:

a) une exploration théorique des thématiques concernant la lecture et l'écriture en deux ou plusieurs langues, et l'interculturalité dans l'enseignement des langues étrangères et secondes.

b) la rédaction de textes de réflexion basés sur la compréhension des processus d'écriture, d'un point de vue personnel et du point de vue de l'enseignement.

4. La production de documents audio et vidéo montrant l'adaptation des activités.

19 Fait de " comprendre la langue de l'autre et se faire comprendre " que ce soit à l'oral ou à l'écrit, (Degache, 2006) cité par Berthele, R. \& Lambelet (2009). La base de ce paradigme est la compréhension de langues typologiquement proches où les compétences partielles, plus particulièrement, les compétences réceptives sont essentielles car, elles permettent de développer l'intercompréhension. 
Les étudiants de master se sont inspirés des activités étudiées au cours du projet dans le but de les faire correspondre (au mieux) aux contextes éducatifs (français ou colombien) dans lesquels ils intervenaient ou étaient susceptibles d'intervenir régulièrement. Leurs productions comprenaient l'adaptation de bandes dessinées, des extraits d'une pièce de théâtre ou d'un roman, la transposition didactique-pédagogique des notions, etc.

Dans cette phase exploratoire, l'étude des premières données permet une caractérisation socioéducative des institutions et des individus, l'établissement d'une analyse de besoins et la perception de processus d'interculturalité.

Le schéma présenté ci-dessous tente de rendre compte du processus itératif et cyclique de la recherche-action-collaborative que nous avons menée et qui s'appuie également sur des principes de théorisation ancrée:

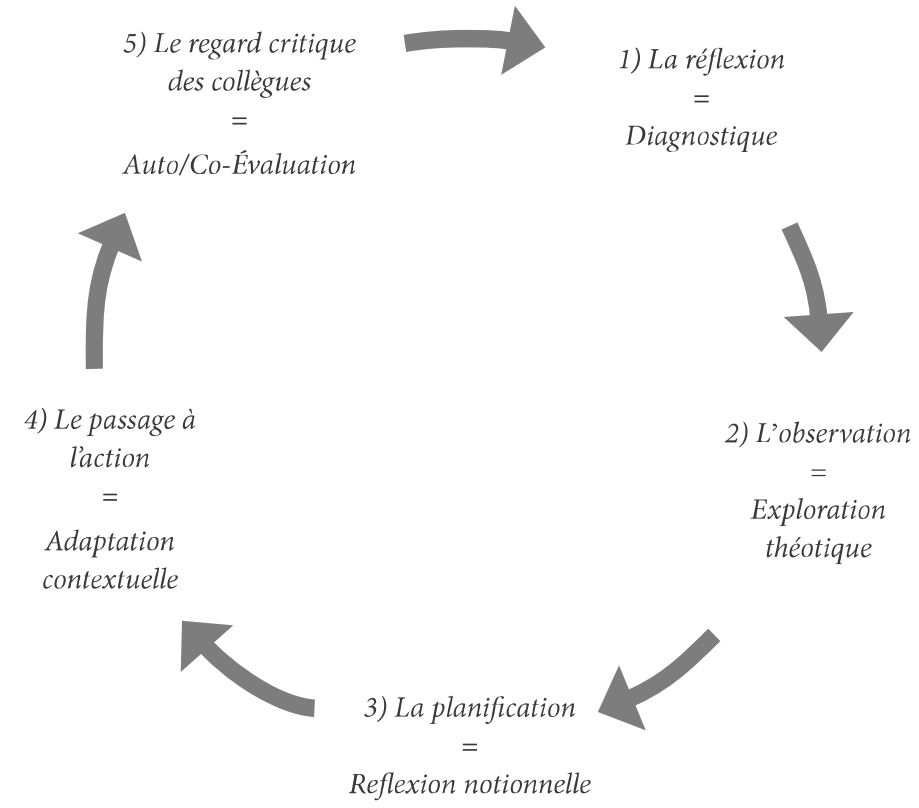

Figure 2. Première phase de notre projet de recherche action Source : création des auteures.

Comme on le voit ici, notre processus de recherche-action collaborative est basé sur la comparaison, le croisement des regards et un agir coopératif (Baudrit, 2007) dans la formation des enseignants.

A partir du processus cyclique illustré par la Figure 2, les questions qui guident notre étude sont: 
- Comment se verbalisent les perceptions et les attitudes de la part des enseignants et des étudiants face à l'altérité dans les deux contextes marqués par variations linguistiques, identités plurielles et métissages, répertoires plurilingues et par une pluralité omniprésente dans - et en dehors de - la classe de langue(s).

- Dès lors, comment les amener à didactiser la pluralité?

\section{Premières observations}

La construction des observables (Blanchet, 2011) est conduite via l'analyse

a. De contenus (Bardin, 1986) et la théorisation ancrée, préalablement systématisés à l'aide du logiciel ATLAs.ti

b. De discursivités, dans la perspective de Maingueneau (2015).

Les 3 macro-catégories thématiques émergentes sont

1. Interculturalité, perceptions et attitudes;

2. Pratiques d'enseignement-apprentissage réflexives;

3. Didactisation.

Les 16 sous catégories résultent de l'analyse des extraits des documents multimodaux qui concentrent 11 types de textes audio-visuels et écrits. Dans cette section, nous analysons des extraits correspondants aux étapes de réflexion, observation et planification du cycle de notre projet de recherche action et aux macro-catégories. Dans cette partie nous présentons les macro-catégories 2 et 3 de façon fusionnée.

\section{Interculturalité, perceptions et attitudes}

Nos premiers constats concernent les perceptions et attitudes que les étudiants associent au terme interculturel: définition personnelle, aspects communs, aspects différents, association à d'autres termes, autres définitions-propositions personnelles, etc.

La perception de l'interculturalité est plus ou moins homogène dans le groupe d'étudiants-enseignants en Colombie et plutôt variée pour le groupe d'étudiants-enseignants en France. Dans le premier contexte, le terme interculturel est initialement présenté comme synonyme de «interacción ou 
interrelación » entre personnes de pays différents. Ensuite, cette interaction ou interrelation est également envisagée entre des personnes appartenant à une même ville, région ou pays. Dans le contexte français, l'interculturel est initialement décrit comme une relation entre plusieurs cultures ou institutions, (au sein de l'école ou dans la société). Ultérieurement, cette description inclue la prise de conscience de sa propre culture ou l'explicitation de méthodes d'apprentissage-enseignement.

Une explication possible de cette différence de perception peut être liée aux périodes d'expositions au terme interculturel. Alors qu'en France, les dimensions interculturelles sont abordées depuis les années 1970 (Barthelemy, Groux \& Porcher, 2011), en Colombie, l'interculturel est particulièrement relié à la reconnaissance et à la défense des minorités linguistiques et " ethniques », et ce, depuis la constitution de 1991. Ce n'est que depuis les années 2000, que le terme s'associe plus à l'enseignement des langues et des cultures étrangères et secondes (Galindo Martínez, Loaiza Villalba \& Botero Restrepo, 2013).

Voici à titre d'exemples, quelques définitions initiales de la notion d'interculturalité proposées par des étudiant(e)s de master:

a. En Colombie:

- "Interacción entre personas de diferentes culturas. Intercambio de saberes, costumbres y experiencias." E1S1C ${ }^{20}$

- "Interrelación de factores propios de una región o país con personas que viven en otras regiones o países.” E2s1C

- "Comprende diferentes habilidades y competencias adquiridas para lograr comprender, valorar e incluso interactuar en diferentes contextos con modos de vida y puntos de vista que atienden a culturas distintas a aquella en la que un sujeto se desenvuelve normalmente." E3S1C

b. En France:

- «La mise en relation de plusieurs cultures, passant par le contact et la connaissance mutuelle. » E1s1F

- «Les relations internationales, le respect de la diversité. Peut se faire dans la politique, dans une école, dans la société en général, à plusieurs niveaux. » E2S1F

- «La relation qu'il peut y avoir entre deux (ou plusieurs) cultures différentes. » E3S1F

Et voici quelques exemples de définitions finales de la notion d'interculturalité proposées par ces mêmes étudiant(e)s: 
a. En Colombie:

- “... lo intercultural también tiene que ver con lo individual y grupal ... con las formas de pensar y de ver el mundo que tiene cada persona y cada grupo de personas en particular y las relaciones que establezcan entre ellos." E1S1C

- “... no incluía elementos como la identidad ni la 'literacidad', entre otros." E2S1C

- “...no solo permite la interacción, sino la aceptación de la diversidad cultural que existe entre unos y otros sin necesidad de ser de diferentes países o regiones. Es comprender e interpretar todo lo que rodea el universo de cada uno y todo lo que influye (familia, colegio, región, amigos, lenguaje, etc.) para comportarnos de algún modo. Un ejemplo es el caso de dos estudiantes que pertenecen a la misma región." E3S1C

b. En France:

- "L'interculturalitéce n'est pas seulement rencontrer une autre culture mais c'est avant tout prendre conscience de celle qui nous est propre." E1S1F

- "J'ai vu qu'au niveau culturel la littérature, les écrits peuvent avoir un rôle plus important que je ne le pensais pas dans l'interculturalité.» E2S1F

- "L'interculturel passe également par l'explicitation des méthodes, par l'écoute de l'autre, pour l'éducation, l'apprentissage de chaque apprenant. » $\mathrm{E} 3 \mathrm{~S} 1 \mathrm{~F}$

On observe que les étudiant(e)s complètent leurs définitions théoriques initiales en intégrant des aspects beaucoup plus proches de leur expérience personnelle.

Tableau 3. Regard croisé sur l'interculturalité

\begin{tabular}{|c|c|}
\hline France & Colombia \\
\hline Relation / Les relations / Se lier avec & Interacción / Interrelación \\
\hline $\begin{array}{l}\text { 1. Prise de conscience de ce qui nous est } \\
\text { propre. }\end{array}$ & $\begin{array}{l}\text { 1. Asociación de lo individual y grupal con } \\
\text { las formas de pensar y de ver el mundo que } \\
\text { tiene cada persona. }\end{array}$ \\
\hline $\begin{array}{l}\text { 2. Association des processus de lecture et } \\
\text { d'écriture à l'interculturalité. }\end{array}$ & $\begin{array}{l}\text { 2. Reconocimiento de elementos como la } \\
\text { identidad o la 'literacidad'. }\end{array}$ \\
\hline $\begin{array}{l}\text { 3. Explicitation des activités/méthodologies } \\
\text { d'enseignement-apprentissage mettant en } \\
\text { avant l'interculturalité. }\end{array}$ & 3. Aceptación de la diversidad cultural. \\
\hline
\end{tabular}


Par ailleurs, les étudiants des deux pays perçoivent de façon plutôt homogène le terme interculturel comme n'étant pas associé à l'idée de conflit, de stéréotype ou d'ethnocentrisme. Leur vision de "relations humaines harmonieuses", (Blanchet \& Coste, 2010, p. 9) proche de celle véhiculée par le Conseil de l'Europe (2008) est associée aux notions de culture individuelle et collective, pluriculturalité, identité, diversité, altérité.

\section{Pratiques d'enseignement-apprentissage réflexives et didactisation}

Nos deux macro-catégories d'analyse suivantes (pratiques d'enseignementapprentissage réflexives et didactisation) sont mises en évidence grâce à l'analyse d'activités d'exploration théorique et de planification méthodologique menées dans les deux cours: en France, " écriture réflexive en didactique des langues et des cultures » et en Colombie, « lectura y escritura en dos lenguas-Biliteracy». Dans ces deux cadres, les masterants ont proposé des activités relevant d'une pédagogie interculturelle que nous synthétisons dans le tableau 4, ci-après.

Tableau 4. Mise en miroir des propositions de didactisation

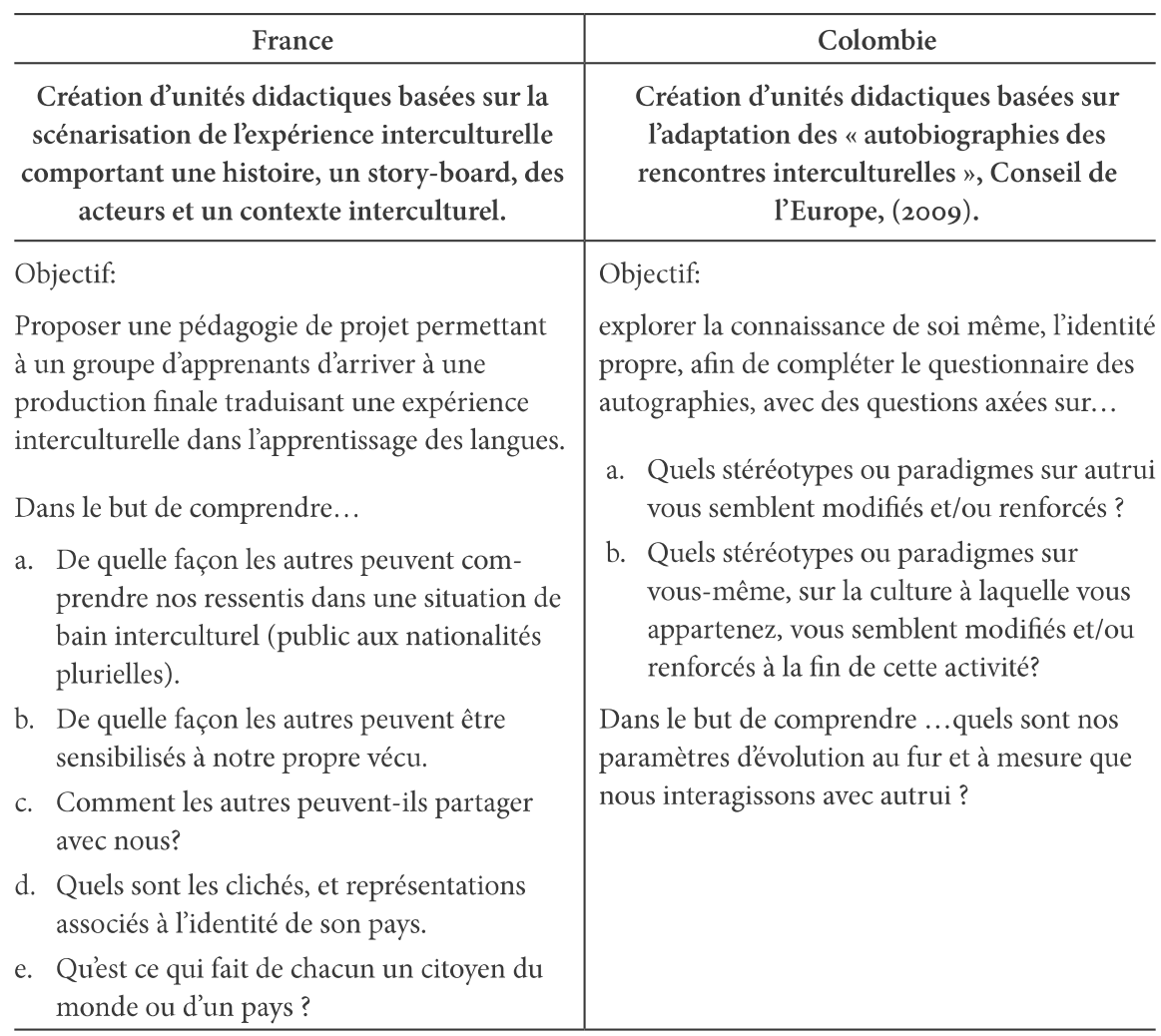

Source : création des auteures. 
Bien qu'il s'agisse de langues d'enseignement et de cultures éducatives différentes, il est possible d'observer des intérêts éducatifs communs:

- L'explicitation des sentiments qui entrent en jeu dans une situation de contact avec l'altérité (rencontre, relation interculturelle) ;

- La reconnaissance des identités de chacun / chacune(e);

- La proposition d'activités originales et créatrices.

Les éléments synthétisés dans le tableau 4 ci-dessus, expriment des processus d'autonomisation et d'empowerment (Le Bossé et Lavallée, 1993), ils signalent qu'une réflexion est en cours sur un enseignement des langues (étrangères, secondes et maternelles) qui prend en compte les réalités sociales des apprenants.

On peut espérer que tels processus mènent:

- À la déconstruction des postures égocentriques;

- À l'élaboration de processus d'auto-conscientisation;

- À la disposition à agir, motivée par une exploration et une sensibilisation conceptuelle ;

- À la perception des opportunités d'action génératrices de propositions de didactisation, en vue d'une circulation entre théorie, méthodologie, et mise en pratique des savoirs.

Du point de vue didactique, les activités mises en place tout au long de cette première étape correspondent au savoir-mettre en relation et à la réflexivité critique. Il s'agit de savoirs où l'altérité, l'interculturalité et les dimensions interculturelles ont une valeur démonstrative (et non pas discursive), d'explicitation de la démarche pédagogique et de recherch; qui mène potentiellement à des changements / transformations dans les paradigmes d'enseignement (voir section Méthodologie Recherche - Action):

Apprendre à voir, à écouter, à être attentif à autrui, apprendre la vigilance et l'ouverture dans une perspective de diversité et non de différences renvoient à la reconnaissance et à l'expérience de l'altérité, expérience qui s'acquiert et se travaille. Autrui ne se laisse pas saisir en dehors d'une communication et d'un échange. Les singularités vulgarisées à tort sous le vocable de différences, sont plus directement perceptibles que l'universalité qui nécessite analyse. (Abdallah-Pretceille, 2006, p. 51).

Notre démarche est présentée comme une variable complémentaire des processus de formations des enseignants / formateurs où l'abordage de la 
culture s'effectue principalement par le biais du sujet, dans les interactions, en contexte et dans l'opposition idio-culture/ culture collective, singulier/ universel, homogénéité/hétérogénéité. Les étudiants sont invités à remettre en question ces schémas d'opposition binaire, (Gamboa-Diaz, 2016; Derivry-Plard, 2015; Abdallah-Pretceille, 2006), à les reconsidérer comme les composantes d'un continuum dans lequel ils vont apprendre à se déplacer, en fonctions des circonstances et des personnes en réactualisant en permanence leurs perceptions de l’altérité.

\section{Premiers constats et conclusions préliminaires}

Nous avons souhaité dans cette contribution expliciter les fondements épistémologiques et le cadre théorique de la recherche-action menée en commun afin d'analyser nos premières données et de présenter les premiers résultats. Mais nous souhaitions surtout poser les fondations d'une éthique commune consistant à articuler les champs de la formation et de la recherche en didactique des langues, du plurilinguisme et de l'interculturel (Cicurel \& Spaëth, 2017).

Cette phase exploratoire de notre recherche nous permet de tirer un certain nombre de constat et de répondre à notre première question: Quelles sont les situations d'altérité auxquelles sont confrontés les enseignants et les étudiants dans les deux contextes? Tout d'abord, les masterants en Colombie ont initialement associé les situations d'altérité, (envisagées comme levier de l'interculturalité), à la culture (pratiques communautaires), aux espaces géographiques et aux habilités sociocognitives 'différents'. Puis, dans leur description finale, ils caractérisent cette notion d'altérité comme relative à tout ce qui est pluriel, à 'ce que chacun est susceptible d'être ou d'agir', ou bien, à 'tout ce qui constitue l'univers et que nous avons également en commun'. Pour les masterants en France, la notion d'altérité est au départ en lien plus direct aux avec les cultures ou à la diversité entre cultures différentes. Finalement, une transformation s'opère vers une altérité qui décrit la prise de conscience de ce qui est propre à chacun, des relations non envisagées initialement avec d'autres sujets d'enseignement et des moyens d'accès à d'autres formes d'apprentissage.

En réponse à notre seconde question (Comment se verbalisent les perceptions et les attitudes de la part des enseignants et des étudiants face à l'altérité dans nos deux contextes, marqués par variations linguistiques, identités plurielles et métissages, répertoires plurilingues et par une pluralité omniprésente dans - et en dehors de - la classe de langue(s) ?), on constate que les étudiants ont attribué une valeur importante à l'introspection et à la connaissance de soi-même pour faire face à ces situations d'altérité. Ils verbalisent leurs perceptions et leurs 
attitudes vis-à-vis de l'altérité, premièrement, à partir d'une prise de conscience de la multidirectionnalité de l'interculturalité associée à : dans des contextes divers: dans des pays différents ou dans un même pays, dans des régions différentes ou dans une même région, dans des villes différentes ou dans une même ville, dans un même quartier ou encore, dans la même famille. Deuxièmement, ils intègrent l'interculturalité dans l'enseignement des langues maternelles, étrangères, secondes de façon régulière, en utilisant la pluridisciplinarité, la multimodalité (vidéos, chat, lettres, audio, etc.), la réflexivité et la créativité.

Ces productions réflexives mènent à la remise en question des préconceptions et de notions figées, telles que le rôle de l'enseignant et de l'apprenant ou encore la conception « du » langage et de « la» culture. Ce point de départ nous a permis de mener un exercice de reconnaissance de l'influence des histoires de vie singulières d'une part, sur la compréhension de l'interculturel et, d'autre part, dans l'agir pédagogique. C'est ici que la notion d'interaction au sens large, c'est-à-dire d'une construction/interprétation réciproques de significations (Liddicoat \& Scarino, 2013) devient pertinente. Enfin, les propositions didactiques expérimentées dans les deux groupes (français et colombien) mettent en évidence la nécessité d'intégrer les multilittératies dans l'exercice introspectif. Ces multilittératies entendues dans la perspective élargie décrite plus haut, intègrent aussi bien les émotions, que les visions de soi-même et du monde dans la réflexion et l'action pédagogique.

Ces premiers constats nous conduisent à poursuivre nos analyses de corpus dans la perspective d'apporter des réponses à notre troisième question: comment faire pour didactiser cette pluralité ? En quoi est-il utile pour le (futur) enseignant de la mettre en mots?

\section{Références bibliographiques}

Abdallah-Pretceille, M. (2006). Interculturalism as a paradigm for thinking about diversity. Intercultural Education, 17(5), 475-483. DOI: 10.1080/1467 5980601065764»10.1080/14675980601065764.

Aubert, N. (dir.) (2004). L'Individu Hypermoderne. Paris. France: Erès.

Auger, N., Dervin, F. \& Suomela-Salmi, E. (dir.) (2009). Pour une didactique des imaginaires dans l'enseignement-apprentissage des langues étrangères. Paris: L'harmattan.

Bardin, L. (1986). El análisis de contenido. Madrid: Akal.

Barkhuizen, G. (éd). (2017). Reflections on language teacher identity research. Abingdon, uk: Routledge.

Barthelemy, F. Groux, D. \& Porcher, L. (2011). Cent mots pour léducation comparée. Paris: l'Harmattan. 
Baudrit, A. (2007). Apprentissage coopératif/Apprentissage collaboratif : d'un comparatisme conventionnel à un comparatisme critique. Les Sciences de l'éducation - Pour l'Ėre nouvelle, 40(1), 115-136. DoI: 10.3917/lsdle.401.0115.

Beacco, J. C., Chiss, J. L., Cicurel, F. \& Véronique, D. (dir.). (2005). Les Cultures Éducatives et Linguistiques dans l'Enseignement des Langues. Paris: Presse Universitaie Francaise.

Berthele, R. \& Lambelet, A. (2009). Approche empirique de l'intercompréhension: répertoires, processus et résultats, Lidil, 39, 151-162.

Blanchet, P. (2011). Les principales méthodes et leurs techniques de construction des observables. Guide pour la recherche en didactique des langues. Approches contextualisés. Paris: Éditions des archives contemporaines. Montréal: Agence universitaire de la francophonie.

Blanchet, P. \& Chardenet, P. (2011) (dir.). Guide pour la recherche en didactique des langues et des cultures. Approches contextualisées. Le français à l'université. Montréal: Éditions des archives contemporaines, Agence universitaire de la francophonie. https://hal.archives-ouvertes.fr/hal-o1436588/ document.

Blanchet P. \& Coste D. (dir.) (2010). Regards critiques sur la notion d' "interculturalité ». Pour une didactique de la pluralité linguistique et culturelle. Paris: L'Harmattan.

Blanchet, P. \& Martinez, P. (2010). Pratiques innovantes du plurilinguisme. Éditions des Archives contemporaines.

Bonilla, C. A. \& Tejada-Sánchez, I. (2016). Unanswered questions in Colombia's foreign language education policy. Profile: Issues in Teachers' Professional Development 18 (1), 185-201.

Bourdieu, P. \& Passeron, J. C. (1977). Reproduction in Education, Culture and Society. London: Sage.

British Council (2015). English in Colombia: An examination of policy, perceptions and influencing factors. May 2015. Rapport. Consulté le 21/12/2017. https://www.teachingenglish.org.uk/sites/teacheng/files/English\%2O in $\% 20$ Colombia.pdf.

Burns, A. (2015). Perspectives on Action Research. Cambridge University Press Colombia.

Byram, M., Gribkova, B., Starkey, H. (2002). Developing the intercultural dimension in language teaching: a practical introduction for teachers. Strasbourg, France: Language Policy Division, Directorate of School, Out-of-School and Higher Education, Council of Europe.

Catroux, M. (2002). Introduction à la recherche-action: modalités d'une démarche théorique centrée sur la pratique, Recherche et Pratiques Pédagogiques en Langues de Spécialité [En ligne], Vol. Xxi n ${ }^{\circ} 3 \mid$ 2002, mis en ligne le 16 mars 2014, consulté le 10 août 2017. http://apliut.revues. org/4276; DOI: 10.400o/apliut.4276. 
Charmaz, K. (2006) Constructing Grounded Theory: A Practical Guide through Qualitative Analysis. Thousand Oaks, CA: Sage, 224 p. ISBN 9780761973539.

Chiss, J. L. (2004). La littératie: quelques enjeux d'une réception dans le contexte éducatif et culturel français. In Barré-De Miniac, C., Brissaud, C., Rispail, M. (éds.). La Littéracie (Conceptions Théoriques et Pratiques d'Enseignement de la Lecture-Écriture). Paris: L'Harmattan, Collection Espaces Discursifs.

Cicurel, F. \& Spaëth, V. (2017). Agir éthique en didactique du FLE/FLS. Le FDLM RA $n^{\circ}$ 62. Cle international. FIPF: Paris.

Coste, D. (1999). 1940 à nos jours. Consolidations et ajustements. Le français dans le monde. Recherches et applications, $\mathrm{n}^{\circ} 25,75-95$.

Coste, D., Moore, D. \& Zarate, G. (1997). Compétence plurilingue et pluriculturelle, Strasbourg. (éd), Du Conseil de l'Europe. https://rm.coe. int/168069d29b.

Conseil de l'Europe (2008). Autobiographie de Rencontres Interculturelles (ARI). En ligne, https://www.coe.int/t/DG4/AUTOBIOGRAPHY/AutobiographyTool_fr.asp. Consulté le 11/11/2017.

Cronquist, K. \& Fizsbein, A. (2017). English language learning in Latin America. Rapport. Inter-American Dialogue. https://www.thedialogue.org/event/ english-language-learning-in-latin-america/ Consulté le 27/11/2017.

De Mejía, A. M. (2002). Power, Prestige and Bilingualism: International Perspectives on Elite Bilingual Education. Multilingual Matters.

De Mejía, A. M. (2005). Bilingual education in Colombia: Towards an integrated perspective. In A.-M. de Mejía (éd.), Bilingual Education in South America (pp. 48-64). Multilingual Matters.

De Mejía, A. M. (2013). Bilingual Education in Colombia: The teaching and Learning of Languages and Academic Content Area Knowledge. In C. Abello-Contesse, P. M. Chandler, M. D. López-Jiménez \& R. Chacón-Beltrán (éds.), Bilingual and Multilingual Education in the 21st Century: Building on Experience (Bilingual Education and Bilingualism) (pp. 42-58).

Derivry-Plard, M. (2015). Les Enseignants de Langues dans la Mondialisation. La Guerre des Représentations dans le Champ Linguistique de l'Enseignement. Paris: Archives contemporaines.

Dick, B. (2007). What can grounded theorists and action researchers learn from each other? In Bryant, A., and Charmaz, K. (éds.). The Sage Handbook of Grounded Theory (pp. 370-388). Thousand Oaks, CA: SAGE.

Fandiño-Parra, J., Bermúdez-Jiménez, J. R. \& Lugo-Vásquez, V. E. (2013, February 6). Retos del Programa Nacional de Bilingüismo. Colombia Bilingüe. Educación y Educadores.

Freire, P. (2006). Pédagogie de l’Autonomie: Savoirs Nécessaires à la Pratique Educative. Toulouse: EREs. (Ouvrage traduit et commenté par JeanClaude Régnier de Pedagogie da atonomia). 
Galindo Martínez, A., Loaiza Villalba, N. \& Botero Restrepo, A. (2013). Bilingüismo, biliteracidad y competencia intercultural: enfoque de investigación cualitativo en contexto escolar. Armenia: Kinesis.

Gamboa-Diaz, P. A. (2014). Éducation interculturelle, mobilité internationale et enseignement supérieur: le cas de la Colombie. Thèse de doctorat non publiée. Université Sorbonne Nouvelle-Paris 3.

Gamboa-Diaz, P. A. (2016). Dimension interculturelle de l'enseignement des langues étrangères en Colombie: un besoin pédagogique pour concilier les politiques éducatives nationales et la réalité multilingues du pays. Actes du colloque 18èmes Rencontres Jeunes Chercheurs (RJC 2015). Paris, ED 268, Université Sorbonne. Nouvelle, 11 et 12 juin 2015. https://hal-univ-paris3. archives-ouvertes.fr/hal-o1495144/document.

Gardin, B. (1976). Pour un enseignement du français aux travailleurs immigrés. Langue Française, (29), 3-16.

Giroux, H. (2004). Critical pedagogy and the postmodern/modern divide: Towards a pedagogy of democratization. Teacher Education Quarterly, 31(1), 31-47. http://www.jstor.org/stable/23478412.

Glaser, B. \& Strauss, A. (1967). The Discovery of Grounded Theory: Strategies for Qualitative Research. Chicago: Aldine de Gruyter.

Goody, J. (1968). Les conséquences de la littératie, Pratiques, n 131/132, 31-68.

Gürüz, K. (2011). Higher education and international student mobility in the global knowledge economy. Albany, NY: State University of New York Press.

Harris, R. (2011). Ethnicity. In Simpson, J. (éd). The Routledge Handbook of Applied Linguistics. London and New York: Routledge. Taylor \& Francis Group.

Jaraba, D. \& Arrieta, A. (2012). Etnoenglish: trenzando palabras, historias y proyectos de vida del pueblo zenú. Íkala, Revista de Lenguaje y Cultura, 17(1), 95-104.

Jarvis, P. (2006). Towards a Comprehensive Theory of Human Learning. London, New-York: Routledge: Taylor and Francis Group.

Jenkins, J. (2006). Current perspectives on teaching world Englishes as a lingua franca. TESOL Quarterly, 40 (1), 157-181.

Knobel, M. \& Kalman, J. (2016). Teacher learning, digital technologies and new literacies. In M. Knobel \& J. Kalman (éds.) New literacies and Teacher Learning: Professional Development and the Digital Turn (pp. 1-20). Peter Lang: New York.

Kramsch, C. (2013). Culture in foreign language teaching. Iranian Journal of language Teaching Research, 1 (1),57-78.

Le Bossé, Y. \& Lavallée, M. (1993). Empowerment et psychologie communautaire : aperçu historique et perspectives d'avenir. Cahiers Internationaux de Psychologie Sociale, 18, 7-20. 
Liddicoat, A. J. \& Scarino, A. (2013). Intercultural language teaching and learning. Chichester, West Sussex, UK; Malden, MA: John Wiley \& Sons.

Maingueneau, D. (2015). Discours, études de discours, analyse du discours. Dans Le Langage en Sciences Humaines et Sociales. Paris: Lambert Lucas.

Martínez Sierra, L. M., Naranjo Cuervo, R. C. \& Rojas Curieux, T. (2010). ewa: Comunidad Virtual de Apoyo a los Procesos de Etnoeducación Nasa. Puutxwe'wna dxi'phadenwa'. Popayán: Universidad del Cauca, Colciencias.

Mendonça Dias, C. (2011). La classe d'accueil, de la pratique à la théorie. Chnane-Davin F. (coord.). La classe de langue, entre théorie et pratique, Le langage et l'homme, 46/2, E.M.E Intercommunications, sprl, 2011, p. 111-122.

Mesmin, C. (1993). Les enfants de migrants á l'école - Réussite, Echec. Grenoble: Edition la Pensée Sauvage.

McLaren, P. (1995). Critical pedagogy and predatory culture. Londres, New York: Routledge.

McNiff, J. (2013). Action Research: Principles and Practice. Routledge.

Mignolo, W., Walsh, C. \& García Linera, A. (2006). Interculturalidad, Descolonización del Estado y del Conocimiento/ Interculturality, Descolonization of The State and Knowledge. Buenos Aires: Ediciones del Signo; [Durham]: Globalization and the Humanities Project (Duke University).

Ministerio de Educación Nacional de Colombia (2001). Etnoeducación una política para la diversidad. Al Tablero $\mathrm{n}^{\circ} 3$. Mis en ligne en avril 2001, consulté le 13 août 2017. http://www.mineducacion.gov.co/1621/ article-87223.html.

Ministerio de Educación Nacional de Colombia (2015). Colombia Bilingüe en el simposio "Desafíos para el desarrollo profesional de los educadores". Mis en ligne le 26 mai 2015, consulté le 13 août 2017. http://www.min educacion.gov.co/cvn/1665/w3-article-351002.html.

Moore, D. \& Molinié, M. (2012). Les littératies: une notion en questions (NEQ) en didactique des langues. Recherches en Didactique des Langues et des Cultures: Les Cahiers de l'Acedle, Notion en Questions (NEQ) en Didactique des Langues, les Littératies, 9 (2), 3-14.

Molinié, M. (1993). Vers une approche relationnelle de la communication interculturelle. Doctorat de $3^{\circ}$ cycle. Didactique des langues et des cultures. Sorbonne Nouvelle Paris 3.

Noël, B. (1991). La métacognition. Bruxelles: De Boeck.

Perrenoud, P. (1998). La transposition didactique à partir de pratiques: des savoirs aux compétences. Revue des Sciences de l'Éducation, 24(3), 487-514.

Randall, S. J. (1992). Colombia and the United States. University of Georgia Press. Rojas Curieux, T. \& Barona Becerra, G. (2007). Del dicho al hecho hay mucho trecho o las vicisitudes de la construcción de una sociedad intercultural. Jangwa Pana, 11(1), 27-39. https://doi.org/10.21676/16574923.787. 
Rojas, A. (2011). Gobernar(se) en nombre de la cultura. Interculturalidad y educación para grupos étnicos en Colombia. Revista Colombiana de Antropología, 47 (2), 173-198.

Strauss A. \& Corbin J. (1990) Basics of Qualitative Research: Grounded Theory Pprocedures and Techniques. Newbury Park. Sage Publications.

Taylor, C. (1989). Les sources du moi. La formation de l'identité moderne. Paris: Seuil.

The Organisation for Economic Co-operation and Development (OECD) (2016), Education in Colombia, OeCD Publishing, Paris. En ligne. http:// dx.doi.org/10.1787/9789264250604-en. Consulté le 2 novembre 2017.

Trocme-Fabre, H. (1987). Je pense, donc je sui. Paris: Editions d'Organisation.

Truscott de Mejía, A. M., López Mendoza, A. A. \& Peña Dix, B. (éds.). (2011). Bilingüismo en el Contexto Colombiano. Iniciativas y Perspectivas en el Siglo XXI (p. 470). Bogotá: Universidad de los Andes.

United Nations Educational, Scientific and Cultural Organization. (2003). Education in a Multilingual World. Paris: Unesco. http://unesdoc.unesco. org/images/oo12/o01297/129728e.pdf Consulté le 19/11/2017.

Vandenberghe, F. (2006). Réflexivité et modernité. In S. Mesure \& P. Savidan (éds.), Dictionnaire des sciences humaines (pp. 975-977). Paris: PUF.

Vigner, G. (1998). Le français des colonies et des indépendances : pour une histoire du français langue seconde. Histoire de la difussion et de l'enseignement $d u$ français dans le monde. Recherches et applicatons. In W. Frijoff \& A. Reboullet (éds.) (pp. 96-104). Paris: Hachette.

Weil, D. K. \& Kincheloe, J. L. (éd). (2004). Critical Thinking and Learning: An Encyclopedia for Parents and Teachers. Westport, Connecticut: Greenwood Press.

Williams, E. (2006). Literacy studies. In A. Davies \& C. Elder. The handbook of applied linguistics (pp. 576-503). Malden, MA: Blackwell Pub. 